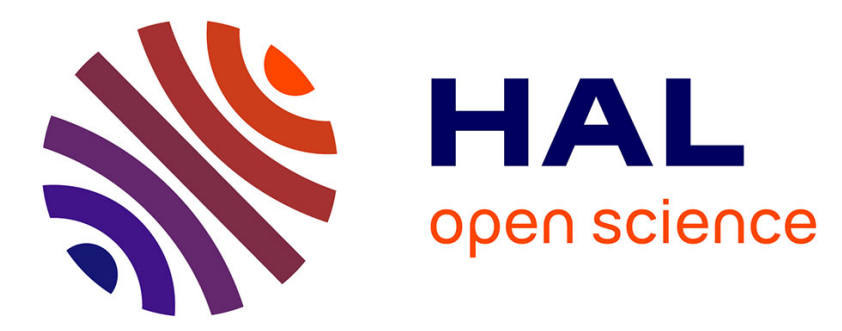

\title{
Optimizing the investments in mobile networks and subscriber migrations for a telecommunication operator
}

Adrien Cambier, Matthieu Chardy, Rosa Figueiredo, Adam Ouorou, Michael Poss

\section{- To cite this version:}

Adrien Cambier, Matthieu Chardy, Rosa Figueiredo, Adam Ouorou, Michael Poss. Optimizing the investments in mobile networks and subscriber migrations for a telecommunication operator. Networks, 2021, 77 (4), pp.495-519. 10.1002/net.21970 . hal-02019853v4

\section{HAL Id: hal-02019853 \\ https://hal.science/hal-02019853v4}

Submitted on 8 Jul 2020

HAL is a multi-disciplinary open access archive for the deposit and dissemination of scientific research documents, whether they are published or not. The documents may come from teaching and research institutions in France or abroad, or from public or private research centers.
L'archive ouverte pluridisciplinaire HAL, est destinée au dépôt et à la diffusion de documents scientifiques de niveau recherche, publiés ou non, émanant des établissements d'enseignement et de recherche français ou étrangers, des laboratoires publics ou privés. 


\title{
Optimizing the investments in mobile networks and subscriber migrations for a telecommunication operator
}

\author{
Adrien Cambier ${ }^{1,2}$ | Matthieu Chardy ${ }^{1}$ | Rosa \\ Figueiredo $^{2}$ | Adam Ouorou ${ }^{1}$ | Michael Poss ${ }^{3}$
}

${ }^{1}$ Orange Labs, 92320 Châtillon, France

${ }^{2}$ LIA, Avignon Université, Avignon, France.

${ }^{3}$ UMR CNRS 5506 LIRMM, Université de Montpellier, Montpellier, France.

\section{Correspondence}

Adrien CAMBIER, Orange Labs, 44 avenue de la République, 92320 Châtillon, France

Email: adrien.cambier@orange.com

Funding information
We consider the context of a telecommunication company that is at the same time an infrastructure operator and a service provider. When planning its network expansion, the company can leverage over its knowledge of the subscriber dynamic to better optimize the network dimensioning, therefore avoiding unnecessary costs. In this work, the network expansion represents the deployment and/or reinforcement of several technologies (e.g., 2G, 3G, 4G), assuming that subscribers to a given technology can be served by this technology or older ones. The operator can influence subscriber dynamic by subsidies. The planning is made over a discretized time horizon while some strategic guideline requirements are required at the end of the time horizon. Following classical models, we consider that the willingness of customers for shifting to a new technology follows an $S$-shape piecewise 
constant function. We propose a MixedInteger Linear Programming formulation, improved through several valid inequalities and a heuristic algorithm. We assess the formulation numerically on real instances.

\section{KEYWORDS}

OR in telecommunications, Capacity Expansion, Investments Optimization, Subscriber Dynamic, Bass model, Mixed-Integer Linear Programming

\section{1 | INTRODUCTION}

Over the last few years, new bandwidth-consuming usages such as video streaming (see Table 1 . have appeared, increasing the average monthly consumption by user, known as Average Usage per User. This phenomenon, correlated with an increase in the number of users, induces natural traffic growth. According to the Visual Networking Index of the IT and network company CISCO [5], traffic will globally reach 49 Exabytes per month in 2020 with a compound annual growth rate of $47 \%$, this growth being particularly important in Africa (65\%). Network expansion is necessary to support such traffic growth.

\begin{tabular}{|c|c|c|}
\hline Service & 2015 & 2018 \\
\hline Youtube videos viewed & $2.78 \mathrm{M}$ & $4.3 \mathrm{M}$ \\
\hline Netflix hours watched & 69444 & 266000 \\
\hline Instagram scrolling & 38000 & 174000 \\
\hline
\end{tabular}

TAB LE 1 Number of usages of some services happening worldwide on the internet in 60 seconds (from 6]).

Whenever possible, telecommunication companies must hence satisfy the request of subscribers in speed and volume to remain competitive, which requires network investments (several billion $€$ to improve the mobile network in the last six years, see [18]). Facing both needs of offering a satisfying service and of limiting the investments, the operator does not want to under-/over- dimension its network.

In many countries, telecommunication companies are both infrastructure operators and service providers. As infrastructure operators, these companies are responsible for planning their network expansion. As service providers, they design the offers for users and have an influence on network traffic. In this manuscript, we consider a model where each offer can be characterized by the technology that its subscribers can reach. This model will simplify the notation used throughout since 
offers and technologies are in bijection from the subscriber viewpoint. The network expansion decisions can benefit from taking into account the subscriber dynamic, and vice-versa. Thus, operators wish to understand the willingness of subscribers to shift to a new technology in order to optimally plan the investments in new mobile generations.

Having a deep and rigorous analysis of the demand evolution can be an advantage for an operator compared to others. This enables the operator to plan financial subsidies, e.g., cost reduction on a phone having access to the newest generation, in order to manage the network expansion and its market share.

\begin{tabular}{|c|c|c|c|c|c|}
\hline & $1 \mathrm{G}$ & $2 \mathrm{G}$ & $3 \mathrm{G}$ & $4 \mathrm{G}$ & $5 \mathrm{G}$ \\
\hline Date & $1980 \mathrm{~s}$ & 1990 & 2003 & 2009 & 2020 \\
\hline Speed & $2.4 \mathrm{~KB} / \mathrm{s}$ & $64 \mathrm{~KB} / \mathrm{s}$ & $2 \mathrm{MB} / \mathrm{s}$ & $1 \mathrm{~GB} / \mathrm{s}$ & $>1 \mathrm{~GB} / \mathrm{s}$ \\
\hline
\end{tabular}

TABLE 2 Evolution of speed through mobile generations (from [20]).

Planning the network expansion is a process that is inherently multi-period (see Remark 13. since investments must be distributed along a couple of years. As often in such problems, it is more efficient to use a strategy that considers multiple years simultaneously. This is even more important in mobile networks because of the quick progress of mobile technology. This is illustrated in Table 2 that shows the speed increase through mobile generations. According to the 2020 annual report of the GSM 1 Association (see [11] for the detailed report), 4G has become the leading mobile network technology worldwide by number of connections (more than 3 billion and $52 \%$ of the total number of connections) in 2019. In this report, the GSM Association forecasts that 4G technology will peak at $60 \%$ of connections in 2023 , while $5 \mathrm{G}$, already launched in 24 countries, will account for $20 \%$ of global connections in 2025.

This fast roll-out of mobile generations leads to a cyclic dependency between the subscriber and the network dynamics as investments in the network promote new subscriptions which in turn lead to new investments.

As we could expect, the network dynamic adds important constraints that must be considered when planning investments related to new mobile generations. For example, dismantling one generation of a mobile network is not an easy option since operational teams are reluctant to abandon well-functioning (and robust) technologies for new ones without back-up. Moreover, several services may need old(er) technologies (machine-2-machine, roaming, ...). Hence, different technologies have to co-exist and operators have to maintain simultaneously several generations.

\section{1 | Mobile Master Plan}

The points raised above motivate us to study the design of multi-period master plans for mobile networks (Mobile Master Plans), which consists in deciding, for a given set of time points and in a given

${ }^{1}$ GSM Association is an originally-European trade body that represents the interests of mobile network operators worldwide. 
area served by telecommunication sites, how to invest in the evolution of network technologies regarding three aspects: densification, sites coverage extension and subscription upgrades. An investment in densification means adding new pieces of equipment (modules) of a given technology in order to increase the capacity of a site already covered by this technology. An investment in coverage extension means the deployment of a given technology on telecommunication sites not covered so far by this technology. Finally, an investment in user upgrades means the offer of financial subsidies to the subscribers in order to promote upgrades from their current subscription to the newest technology available. As already mentioned, these three kinds of investment decisions must be synchronized.

In order to be well-placed in operator rankings [1], strategic guidelines are decided by the operator at the end of the time horizon and drive the investments in order to guarantee competitiveness (for instance: sites coverage, user coverage, experienced throughput). In practice, Mobile Master Plans are designed for a 5-year time horizon with decisions taken for each year in this period. Mobile Master Plans are driven by cost minimization while ensuring strategic targets over the whole time horizon.

\section{2 | Related literature}

In this work, we are interested in optimizing, over a time horizon, investment decisions related to network expansion and subscriber dynamics assuming the arrival of a new technology. Such problems have been treated in the literature but with other targets in mind and a focus on network investments.

\subsection{1 | Network expansion}

Capacity expansion problems in telecommunication networks have been studied for a long time in the integer programming community, see [13, 10 among many others, including the case of multiperiod planning [8, 9 , 14].

Closer to the context of mobile capacity expansion, the authors of [4 present an exact mixedinteger formulation and a heuristic method to compute a Mobile Master Plan in a restrictive framework. These models integrate the upgrade of subscribers thanks to subsidies. However, a limitation of the models studied in 4 is that the amount of subsidy offered to users is fixed and the number of users which upgrade is set as a variable, constrained only to be positive and upper bounded by the total number of users. The possibility of offering subsidies in order to increase user upgrades is hence not taken into account. In [15], the authors consider the transition of a generation to another, but from a subscriber migration point of view only, applied for a South Korean network.

\subsection{2 | The Bass model}

Our Mobile Master Plan shall offer financial subsidies to the customers to encourage them to shift to the newest technology. To model the effect of these subsidies, we rely on the well-known Bass 
model from the marketing literature.

First studies on diffusion of innovation and new products appeared in the 60's, in a period of high economic growth and important innovations (television, etc). Everett Rogers published the diffusion of innovation theory in 1962 (see [19), based on the adoption curve of Figure1]

This curve presents the percentage of subscribers which adopt a new product during the time horizon. The curve assumes that the timing of a consumer's initial purchase is related to the number of previous buyers (imitation part) and enlightens different types of subscribers. The innovators are the easiest to convince and the laggards are those who adopt the last. External factors (marketing and attractiveness) due to decisions taken in the time horizon are not taken into account, as well as the generation effect (new generation replacing an older one).

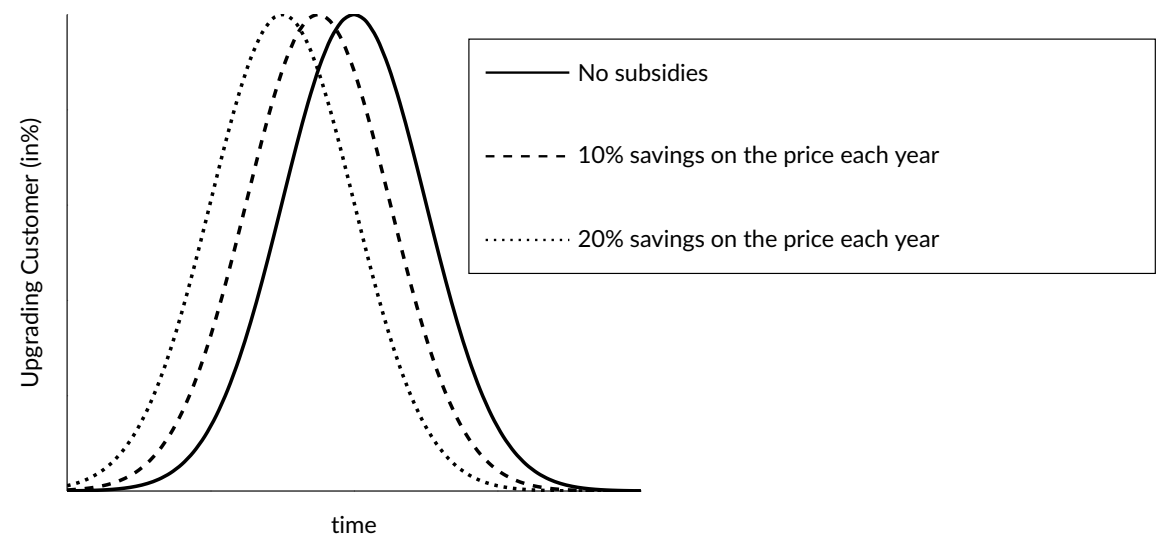

FIGURE 1 Curve of the diffusion of innovation and influence of subsidies.

In 1969, Bass formalized Roger's model by using differential equations and later developed it to tackle some of the issues with external variables (see [2]) and generations (see [17]). This work considers marketing aspects by showing a left shift on the shape of adoptions curves when regular savings (equivalent to constant subsidies) on the price of a product are made, as illustrated in Figure 1 These models help the understanding of how subscribers react in a telecommunication market: see Section 5 in [3] for a discussion on 2G/3G upgrade; [15] for Bass model applied to the forecasting of the $5 \mathrm{G}$ upgrade; and [16] for an application to the Greek mobile market.

In order to adapt this formalism to the current telecommunication context, we consider two important factors for modeling the adoption of a new technology, described hereafter by function $f$. First, the percentage of subscribers shifting from older technologies is very sensitive to the price gap between the new technology and their current one, which will be referred to as $\sigma$. The second factor is the influence of network deployment (denoted $c$ ). Indeed, subscribers upgrade more easily when they are sure to benefit from the new service, i.e. if the newest technology is deployed. 


\subsection{Contributions and structure of the paper}

To the best of our knowledge, jointly optimizing the two dynamics (network and subscribers) under capacity and targeting constraints has not yet been studied. Hence, the first main contribution of the manuscript is to provide a realistic model for the problem. Building on practical considerations, we come up with a non-linear non-convex Mixed-Integer Programming (MIP) formulation, the nonlinearity of which comes from the aforementioned function $f$. Practical considerations will imply that the domain of $f$ is finite, making $f$ a discrete function. Hence, the linearization of the model will naturally follow using classical techniques.

The second main contribution of the manuscript lies in improving the performance of the model through (i) the strengthening of the MILP with several families of valid inequalities, and (ii) a heuristic algorithm that assigns fixed values of the decision variables (subsidies and coverage) and solves the resulting problem as a knapsack problem.

Our third contribution lies in assessing in details the performance and solutions provided by the model on real instances. Our results show, in particular, the efficiency of the valid inequalities and the heuristic algorithm, enabling us to solve large real-life instances to near-optimality. We also assess scalability performances and economic costs when is introduced a smoothing constraint stating that the operator does not want high budget discrepancy over the years.

The remainder of this article is organized as follows. Section 2 introduces our Mobile Master Plan problem for two technologies, for which a mixed integer formulation is provided and linearized in Section 3 Section 4 introduces the aforementioned valid inequalities and heuristic algorithm. These models are numerically assessed in Section 5 on real-life instances. Concluding remarks are given in Section 6

\section{2 | PROBLEM DESCRIPTION}

The time horizon is taken as multi-period with equally-sized time periods denoted by $t \in \mathcal{T}=$ $\{1, \ldots, \bar{t}\}$ (typically 5 periods of one year each). We add "0" for denoting the beginning of the time horizon.

The whole area is served by existing telecommunication sites potentially equipped with at most two mobile network technologies. We thus consider a set $\mathcal{G}=\{C G, N G\}$ of mobile network generations (the current one and the newest one being deployed) and a set $\mathcal{S}=\left\{1, \ldots, N_{S}\right\}$ of telecommunication sites. As we have already mentioned, the network capacity on a site can be increased in two ways: by deploying a technology on a site or by adding new modules of an already deployed technology. In this work, we assume that the current technology $C G$ is deployed on all sites at the beginning of the time horizon and that only the newest technology $N G$ can be deployed during the time horizon with a cost per site of $C A_{N G}$. The binary parameter $Z_{s, N G}^{0}, s \in \mathcal{S}$, is equal to 1 iff the newest network technology $N G$ is deployed on site $s$ at the beginning of the time horizon. The initial number of modules on each site is denoted by $M_{s, g}^{0}, s \in \mathcal{S}, g \in \mathcal{G}$. For each site and for each deployed technology, adding new modules is possible with a unitary cost of $C M_{g}, g \in \mathcal{G}$. Note that we consider that network costs do not vary over the time horizon as, in an operational context, 
these are settled through long-time procurement with suppliers (covering the network life-cycle). The network decommissioning (possibility of removing modules) is linked to spectral considerations and hence is outside of the scope of this article. Technical constraints impose an upper bound on the number of modules that can be added to a site for each technology, which we note $\bar{M}_{g}, g \in \mathcal{G}$. Let us introduce $C A P_{g}, g \in \mathcal{G}$, the unitary capacity of a module of each network technology.

The initial number of subscribers to each technology associated with each site is denoted by $U_{s, g}^{0}, s \in \mathcal{S}, g \in \mathcal{G}$. These values are the values used for the network dimensioning, following usual rules (see dimensionning rules below).

For technical reasons, subscribers cannot be served by a more recent technology than the one they subscribe to. Hence, $C G$ subscribers have to be served by $C G$ technology. For quality of experience motivations, we introduce a load-balancing rule stating that $N G$ subscribers associated to a site $s \in \mathcal{S}$ are served by $N G$ technology if deployed on $s$ and $C G$ technology otherwise.

As mentioned in the introduction, we assume in this work that the whole investments in user upgrades are made towards the newest technology NG. We also assume that the upgrade mechanism modeling subscribers willing to shift to $N G$ technology depends only on two parameters. The first one is the value of the subsidy denoted by $\sigma$. The set of possible values taken by $\sigma$ will be denoted by $\mathcal{K}$. This set is finite due to practical considerations (modeling traditionally used marketing offers : $20 \%, 30 \%$ savings on the new phone etc). The second one is an indicator of the level of $N G$ technology deployment. This indicator will be taken as a range of coverage $c$ (low, medium low, medium high and high coverages for instance). The range of coverage of a given time period will be measured as the range of the proportion of sites on which $N G$ technology is deployed, denoted $\alpha$ and referred as sites coverage, in what follows. For modeling coverage ranges, we partition the interval $[0,1]$ into $C$ smaller intervals $\left[L_{c}, U_{c}[\right.$, and define $C=\{1, \ldots, C\}$. The function modeling the upgrade mechanism, denoted by $f: \mathcal{K} \times C \rightarrow[0,1]$, provides the proportion of subscribers willing to shift to $N G$ technology if they receive the given subsidy $\sigma \in \mathcal{K}$ under a given range of coverage $c \in C$ at the beginning of the time period $t \in \mathcal{T}$. This function is assumed non-decreasing in both arguments. We denote by $N_{g}^{t}, t \in \mathcal{T}, g \in \mathcal{G}$ the forecasted percentage of incoming users subscribing to technology $g$ in time period $t$. New subscribers are assumed to be assigned to sites proportionally to the total number of subscribers at the end of the previous time period.

As already pointed out, network and subscriber dynamics are linked. First, each network generation has to be dimensioned to handle the traffic demand per subscriber $D_{o, g}^{t}, t \in \mathcal{T}, o \in \mathcal{G} g \in \mathcal{G}$. These demands are forecasted Average Usage per User, converted in traffic demand by usual dimensioning rules (5\% of monthly traffic happens during the 22 busiest hours of the month) - see 12 . As $C G$ subscribers are only served by $C G$, we simplify notations by stating that a $C G$ subscriber will have the demand $D_{C G}^{t}$. An NG subscriber will have the demand $D_{N G, N G}^{t}$ if served by $N G$ and $D_{N G, C G}^{t}$ otherwise. We assume that $D_{C G}^{t} \leq D_{N G, C G}^{t}<<D_{N G, N G}^{t}$. Indeed, we consider than the technical limitations on connections make the traffic generated by a $N G$ user on $C G$ far lower than the traffic generated by a $N G$ user on $N G$, and superior or equal to the traffic generated by a $C G$ user. Second, telecommunication operators are ranked according to their performance. Therefore, we decide to focus on requiring satisfying levels for two key performance indicators: the proportion of sites covered by $N G$ at the end of the time horizon, which is denoted by $\alpha^{\bar{t}}$ and the averaged 
quality of experience to the corresponding subscribers. The averaged quality of experience is guaranteed by asking for a minimal proportion of the total number of subscribers being $N G$ subscribers associated with $N G$ sites. These subscribers benefit indeed from the new performance and have the maximum throughput. The thresholds required at the end of the time horizon associated with these two targeting indicators are respectively denoted by $\underline{\alpha}$ and $\underline{Q O E}$.

Decisions are taken over the time horizon. These decisions are the deployment of $N G$ technology, the number of modules added (for all technologies), and the subsidies given to the subscribers from older technologies for upgrading towards $N G$ technology. The problem defined in this work, denoted as the Mobile Master Plan Problem (MMPP), consists in finding the decisions which minimize network and subscribers investments over the time horizon while satisfying capacity and targeting constraints.

A network representation of the MMPP for an example with three sites and two time periods is provided in Figure 2

Parameters introduced in this section are summed up below:

- $C A_{N G}$ is the cost of adding $N G$ technology,

- $C M_{g}$ is the cost of adding a module of a technology $g \in \mathcal{G}$,

- $M_{s, g}^{0}$ stands for the initial number of modules of technology $g \in \mathcal{G}$ on site $s \in \mathcal{S}$,

- $\bar{M}_{g}$ stands a technical upper bound on the number of modules of technology $g \in \mathcal{G}$,

- $Z_{s, N G}^{0}$ stands for the initial presence (yes/no) of $N G$ technology on site $s \in \mathcal{S}$,

- $U_{s, g}^{0}$ is the initial number of subscribers on site $s \in \mathcal{S}$ to technology $g \in \mathcal{G}$,

- $D_{o, g}^{t}$ is the unitary demand of a subscriber to technology $o \in \mathcal{G}$ served by technology $g \in \mathcal{G}$ at time period $t \in \mathcal{T}$,

- $\quad N_{g}^{t}$ is the percentage of incoming user subscribers to technology $g \in \mathcal{G}$ at time period $t \in \mathcal{T}$,

- $C A P_{g}$ is the capacity of adding a module of a technology $g \in \mathcal{G}$,

- $f(\sigma, c)$ is the reaction to the subsidy offered $\sigma \in \mathcal{K}$ under range of coverage interval $c \in \mathcal{C}$,

- $\sigma^{t} \in \mathcal{K}$ is the value of subsidy offered at time period $t \in \mathcal{T}$,

- $L_{c}$ stands for the lower bound of coverage range $c \in C$,

- $U_{c}$ stands for the upper bound of coverage range $c \in C$,

- $\alpha^{0}$ stands for the sites coverage at the beginning of the time horizon,

- $\alpha^{t}$ stands for the sites coverage at the end of time period $t \in \mathcal{T}$,

- $c^{t} \in C$ is the range of coverage of $\alpha^{t-1}$ for each time period $t \in \mathcal{T}$,

- $\quad \underline{\alpha}$ and $Q O E$ are the thresholds fixed as strategic guidelines. 


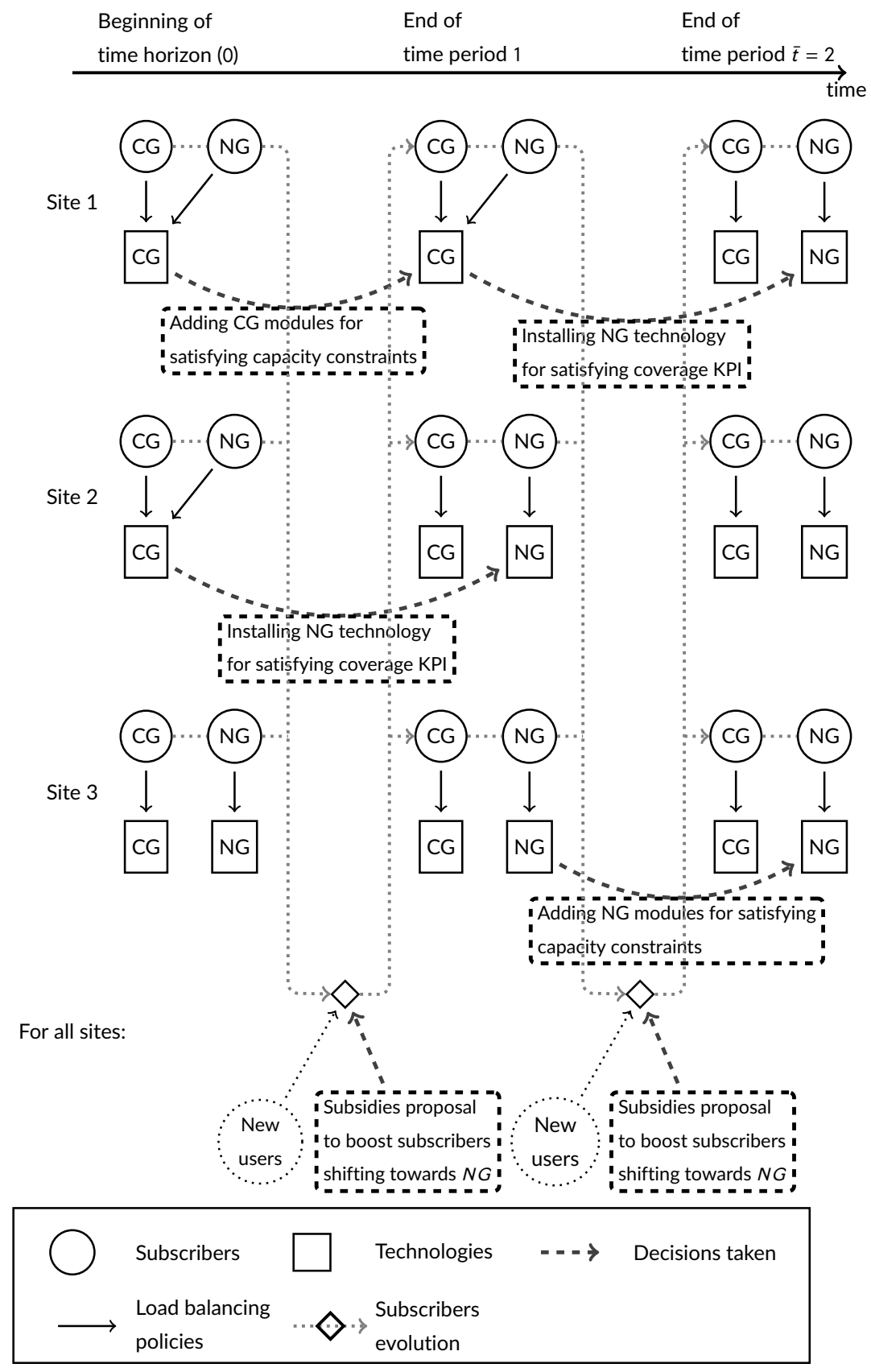

FIGURE 2 Network representation of the MMPP (three sites, two time periods). 


\section{3 | MATHEMATICAL MODELING}

We provide in this section a mixed-integer formulation for the problem described in Section 2 We define the set of decision variables used in our formulation in Section 3.1 and present a non-linear mixed-integer formulation in Section 3.2 Finally, in Section 3.3 we linearize this formulation.

\section{1 | Decision variables}

For modeling the network investment, we use the following variables:

- For $t \in \mathcal{T} \cup\{0\}, s \in \mathcal{S}$, let us introduce the binary variable

$$
z_{s, N G}^{t}=\left\{\begin{array}{l}
1, \text { if the newest technology is deployed at site } s \text { at the end of time period } t \\
0, \text { otherwise. }
\end{array}\right.
$$

- For $t \in \mathcal{T} \cup\{0\}, s \in \mathcal{S}, g \in \mathcal{G}$, the integer variable $m_{s, g}^{t}$ represents the total number of modules of technology $g$ deployed on site $s$ at the end of time period $t$.

As for modeling the number of users on each site, we use the following continuous variables:

- For each $t \in \mathcal{T} \cup\{0\}, s \in \mathcal{S}, g \in \mathcal{G}$, let $u_{s, g}^{t}$ denote the total number of subscribers to technology $g$ in site $s$ at the end of time period $t$ (we denote an upper bound on this quantity by $\bar{U}_{s, g}^{t}$ ),

- For each $t \in \mathcal{T}, s \in \mathcal{S}, o, g \in \mathcal{G}^{2}$, let $u_{s, o, g}^{t}$ denote the total number of subscribers to technology $o$ served by technology $g$ in site $s$ at the end of time period $t$.

In addition, the notation $\sigma^{t}, c^{t}$, and $\alpha^{t}$ introduced in the previous section become optimization variables:

- For each $t \in \mathcal{T}$, let $\sigma^{t}$ be the value of the subsidy, in $\mathrm{k} €$, offered to subscribers to former technologies for upgrading to technology $N G$ at the beginning of time period $t$,

- For each $t \in \mathcal{T}$, let $\alpha^{t}=\frac{\sum_{s \in \mathcal{S}} z_{s, N G}^{t}}{N_{S}}$ be the redundant variable that denotes the $N G$ sites coverage (fraction of sites where $N G$ technology is deployed) at the end of the time period $t$,

- For each $t \in \mathcal{T}$, let $c^{t}$ denote the interval of $C$ to which $\alpha^{t-1}$ belongs.

The upgrade function, representing the percentage of users reacting positively to a subsidy $\sigma \in \mathcal{K}$ for a given coverage $c \in C$, is denoted by $f(\sigma, c)$ and will be modeled explicitly in Section 3.3

Remark 1 The total number of subscribers is constant as it does not depend on the value taken by the optimization variables. We denote this constant by UTOT t for each time period $t$ and each site $s$. It is 
recursively defined as:

$$
\begin{array}{r}
U T O T_{s}^{0}=U_{s, C G}^{0}+U_{s, N G}^{0} \\
U T O T_{s}^{t}=\left(1+N_{C G}^{t}+N_{N G}^{t}\right) U T O T_{s}^{t-1}
\end{array}
$$

\section{2 | General Formulation}

The MMPP can be modeled as follows:

$$
\begin{aligned}
& \min \sum_{t \in \mathcal{T}} \sigma^{t} f\left(\sigma^{t}, c^{t}\right) \sum_{s \in \mathcal{S}} u_{s, C G}^{t-1}+\sum_{s \in \mathcal{S}} \sum_{g \in \mathcal{G}} C M_{g}\left(m_{s, g}^{\bar{t}}-M_{s, g}^{0}\right) \\
& +\sum_{s \in \mathcal{S}} C A_{N G}\left(z_{s, N G}^{\bar{t}}-Z_{s, N G}^{0}\right)
\end{aligned}
$$




$$
\begin{aligned}
& u_{s, o, g}^{t} \geq 0 \\
& \sigma^{t} \in \mathcal{K} \\
& c^{t} \in C
\end{aligned}
$$$$
\forall s \in \mathcal{S}, \forall t \in \mathcal{T}, \forall o, g \in \mathcal{G}^{2},
$$$$
\forall t \in \mathcal{T} \text {, }
$$

We denote this formulation by $\mathcal{M}^{N L}$. The objective function 1 minimizes both subscribers migration costs and network investments. The first term stands for the offered subsidies (user upgrades), the second term for the adding of new modules for increasing the capacity (densification), and the third term for the deployment of the newest technology NG (coverage extension). Constraints 2]4 are the network dynamic constraints. Constraints 2 - 3 define the upper bounds on the numbers of modules for each technology deployed on each site. These constraints also ensure that if a technology is not deployed, no corresponding modules can be added. Constraints 4 prevent from decommissioning by imposing the number of modules of each technology to be non-decreasing during the time horizon.

Constraints [5]-8] are the network dimensioning constraints, in charge of making the link between the network dynamic and the subscriber dynamic. Constraints 5 ensure that on each site, $N G$ subscribers can be served by $C G$ or $N G$ technologies. If $N G$ technology is not installed, they are served by $C G$ technology (see capacity constraints thereafter). Constraints 6 state that they are served by $N G$ technology if it is installed, ensuring the load-balancing rule. Constraints 7 and 8 are the capacity constraints: the installed capacities of each technology on each site have to be sufficient for providing services for all users located at this site and having to be served by this technology. They also ensured the technical incompatibility stating that $C G$ subscribers cannot be served by $N G$ technology.

Constraints 9-10 are the subscriber dynamic constraints. They define the total number of subscribers to $C G$ and $N G$ technologies at each site and each time period, taking into account former $C G$ subscribers who decide to shift to $N G$ technology, thanks to subsidies and coverage improvements. Constraints 11]-12] stand for the model strategic guidelines and refer to the end of the time horizon. Constraint 11] ensures the threshold of subscribers covered by the newest technology is met. The indicator is proportional to the quality of experience which measures the percentage of users having access to the new technology throughput. Constraint 12, imposes that the threshold on the number of sites on which $N G$ is deployed is met. Constraints 13 stand for defining variables $\alpha^{t}$. Constraints 14 define the range of coverage used in the subsidy function as the range of coverage to which belongs $\alpha^{t-1}$. Constraints 15 - 17 refer to the initial conditions. Finally, constraints 18 - 23. define the domain of the variables. Section 3.3 details function $f$ and linear modeling of constraints [14.

Remark 2 The size of formulation $\mathcal{M}^{N L}$ can be reduced by replacing variables $m_{s, g}^{t}$ for each $t \in \mathcal{T} \backslash\{\bar{t}\}$ by $m_{s, g}^{\bar{t}}$ and removing constraints 4. Indeed variables $m$ are only required to be lowerly and upperly bounded and to be non-decreasing over the time horizon while the objective function only depends on $m_{s, g}^{\bar{t}}$. However, we choose to present a model with variables $m_{s, g}^{t}$ as a generic basis for businesses applications where budget has to be controlled over time, thus requiring the temporal dynamic of the number of modules. We will assess numerically in Section 5 a set of constraints smoothing the costs for the oper- 
ator by bounding budget fluctuations by a percentage $p$. Let the budget spent in each year be denoted by

$$
B_{t}=\sigma^{t} f\left(\sigma^{t}, c^{t}\right) \sum_{s \in \mathcal{S}} u_{s, C G}^{t-1}+\sum_{s \in \mathcal{S}} \sum_{g \in \mathcal{G}} C M_{g}\left(m_{s, g}^{t}-m_{s, g}^{t-1}\right)+\sum_{s \in \mathcal{S}} C A_{N_{G}}\left(z_{s, N G}^{t}-z_{s, N G}^{t-1}\right) \quad \forall t \in \mathcal{T}
$$

so the objective function [1] is actually equal to $\sum_{t \in \mathcal{T}} B_{t}$. The cost equilibrium set of constraints can be written as follows:

$$
(1-p) \times \frac{\sum_{t^{\prime} \in \mathcal{T}} B_{t^{\prime}}}{\bar{t}} \leq B_{t} \leq(1+p) \times \frac{\sum_{t^{\prime} \in \mathcal{T}} B_{t^{\prime}}}{\bar{t}} \quad \forall t \in \mathcal{T} .
$$

With constraints 25 added to formulation $\mathcal{M}^{N L}$, variables $m_{s, g}^{t}$ are no longer redundant.

Remark 3 The time dependency of the technology installation variable $z$ cannot be removed. Indeed, the upgrade reaction at period $t \in \mathcal{T}$ depends on variables $z^{t-1}$ (constraints 13] and 14]). Hence, modifying the period of installation impacts the total upgrade reaction and the objective value. Note that the coverage will not always be set to the highest value due to the subsidies costs.

\subsection{Upgrade function modeling}

As we have mentioned, the upgrade function (function characterizing the proposition of CG subscribers that shift to NG technology) is non-decreasing in both the subsidy amount $\sigma$ and the range of coverage $c$. Figure 3 provides an example with four ranges.

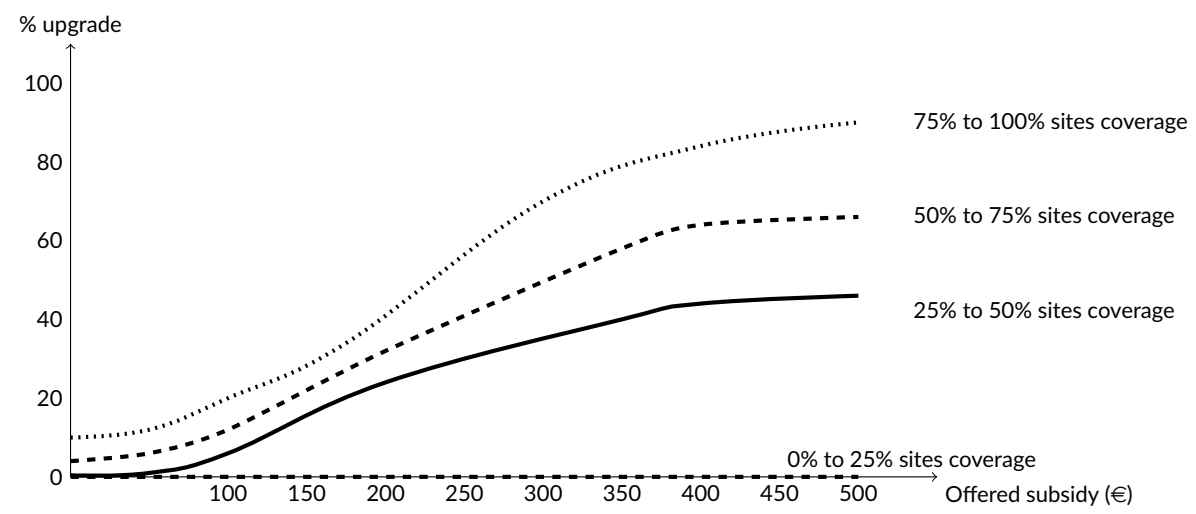

FIGURE 3 Example of upgrade function.

To shorten notation, we denote by $f_{\sigma, c}$ the percentage of subscribers that react positively when subsidy $\sigma \in \mathcal{K}$ is offered and the $N G$ sites coverage belongs to the range $\left[L_{c}, U_{c}[\right.$, formally defined as $f(\sigma, c)$. Aiming to incorporate this in our MILP, we introduce a binary variable $\delta_{\sigma, c}^{t}$ for each 
$t \in \mathcal{T}, \sigma \in \mathcal{K}, c \in \mathcal{C}$, taking value equal to 1 iff $\sigma^{t}$ is offered and $\alpha^{t-1} \in\left[L_{c}, U_{c}[\right.$. The first term of objective function [1] from Section 3.2 can be rewritten as follows:

$$
\sum_{t \in \mathcal{T}} \sum_{\sigma \in \mathcal{K}} \sum_{c \in C} \sigma f_{\sigma, c} \delta_{\sigma, c}^{t} \sum_{s \in \mathcal{S}} u_{s, C G}^{t-1}
$$

Also, constraints 9 and 10 can be written as:

$$
\begin{aligned}
& u_{s, C G}^{t}=u_{s, C G}^{t-1}+N_{C G}^{t} U T O T_{s}^{t-1}-\sum_{\sigma \in \mathcal{K}} \sum_{c \in C} f_{\sigma, c} \delta_{\sigma, c}^{t} u_{s, C G}^{t-1} \quad \forall s \in \mathcal{S} \forall t \in \mathcal{T}, \\
& u_{s, N G}^{t}=u_{s, N G}^{t-1}+N_{N G}^{t} U T O T_{s}^{t-1}+\sum_{\sigma \in \mathcal{K}} \sum_{c \in C} f_{\sigma, c} \delta_{\sigma, c}^{t} u_{s, C G}^{t-1} \quad \forall s \in \mathcal{S} \forall t \in \mathcal{T} .
\end{aligned}
$$

We linearize the products of binary variables $\delta_{\sigma, c}^{t}$ and continuous positive variables $u_{s, C G}^{t-1}$ using a classical method [7]. For denoting this product, we introduce the continuous positive variables $\pi_{\sigma, c, s, C G}^{t}=\delta_{\sigma, c}^{t} u_{s, C G}^{t-1}$ for each $t \in \mathcal{T}, \sigma \in \mathcal{K}, c \in \mathcal{C}, s \in \mathcal{S}$. Consequently, the MMPP can be formulated as the following MILP.

$$
\begin{aligned}
& \min \sum_{t \in \mathcal{T}} \sum_{\sigma \in \mathcal{K}} \sum_{c \in C} \sum_{s \in \mathcal{S}} \sigma f_{\sigma, c} \pi_{\sigma, c, s, C G}^{t}+\sum_{s \in \mathcal{S}} \sum_{g \in \mathcal{G}} C M_{g}\left(m_{s, g}^{\bar{t}}-M_{s, g}^{0}\right) \\
& \quad+\sum_{s \in \mathcal{S}} C A_{N G}\left(z_{s, N G}^{\bar{t}}-Z_{s, N G}^{0}\right)
\end{aligned}
$$

s.t. 2] - 7, 11] - 13, 15 - 17

$$
\begin{array}{ll}
u_{s, C G}^{t}=u_{s, C G}^{t-1}+N_{C G}^{t} U T O T_{s}^{t-1}-\sum_{\sigma \in \mathcal{K}} \sum_{c \in C} f_{\sigma, c} \pi_{\sigma, c, s, C G}^{t} & \forall s \in \mathcal{S}, \forall t \in \mathcal{T}, \\
u_{s, N G}^{t}=u_{s, N G}^{t-1}+N_{N G}^{t} U T O T_{s}^{t-1}+\sum_{\sigma \in \mathcal{K}} \sum_{c \in C} f_{\sigma, c} \pi_{\sigma, c, s, C G}^{t} & \forall s \in \mathcal{S}, \forall t \in \mathcal{T}, \\
\sum_{\sigma \in \mathcal{K}} \sum_{c \in C} \delta_{\sigma, c}^{t}=1 & \forall t \in \mathcal{T}, \\
\sum_{\sigma \in \mathcal{K}} \delta_{\sigma, c}^{t} \leq 1+U_{c}-\alpha^{t-1} & \forall t \in \mathcal{T}, \forall c \in \mathcal{C}, \\
\sum_{\sigma \in \mathcal{K}} \delta_{\sigma, c}^{t} \leq 1+\alpha^{t-1}-L_{c} & \forall t \in \mathcal{T}, \forall c \in \mathcal{C}, \\
\pi_{\sigma, c, s, C G}^{t} \leq \delta_{\sigma, c}^{t} \bar{U}_{s, C G}^{t-1} & \forall s \in \mathcal{S}, \forall t \in \mathcal{T}, \forall \sigma \in \mathcal{K}, \forall c \in \mathcal{C}, \\
\pi_{\sigma, c, s, C G}^{t} \leq u_{s, C G}^{t-1} & \forall s \in \mathcal{S}, \forall t \in \mathcal{T}, \forall \sigma \in \mathcal{K}, \forall c \in C,
\end{array}
$$




$$
\begin{array}{ll}
\pi_{\sigma, c, s, C G}^{t} \geq u_{s, C G}^{t-1}-\left(1-\delta_{\sigma, c}^{t}\right) \bar{U}_{s, C G}^{t-1} & \forall s \in \mathcal{S}, \forall t \in \mathcal{T}, \forall \sigma \in \mathcal{K}, \forall c \in C, \\
m_{s, g}^{t} \in \mathbb{N} & \forall s \in \mathcal{S}, \forall t \in \mathcal{T} \cup\{0\}, \forall g \in \mathcal{G}, \\
z_{s, N G}^{t} \in\{0,1\} & \forall s \in \mathcal{S}, \forall t \in \mathcal{T} \cup\{0\}, \\
u_{s, g}^{t} \geq 0 & \forall s \in \mathcal{S}, \forall t \in \mathcal{T} \cup\{0\}, \forall g \in \mathcal{G}, \\
u_{s, o, g}^{t} \geq 0 & \forall s \in \mathcal{S}, \forall t \in \mathcal{T}, \forall 0, g \in \mathcal{G}^{2}, \\
\delta_{\sigma, c}^{t} \in\{0,1\} & \forall t \in \mathcal{T}, \forall \sigma \in \mathcal{K}, \forall c \in C, \\
\pi_{\sigma, c, s, C G}^{t} \geq 0 & \forall s \in \mathcal{S}, \forall t \in \mathcal{T}, \forall \sigma \in \mathcal{K}, \forall c \in C .
\end{array}
$$

We denote this formulation by $\mathcal{M}$. Constraints 29] and 30] are the linearizations respectively of constraints 26] and 27. Constraints 31 ensure that one and only one subsidy from the set $\mathcal{K}$ is offered at each time period, the case when no subsidy is given being represented by $\sigma=0$. Constraints 32 and 33 ensure that, for each time period, variables $\delta_{\sigma, c}^{t}$ are set according to the coverage. Constraints 32] (respectively [33) set all $\delta$ related to a range to 0 if the coverage is greater (resp. smaller) than the upper (resp. lower) bound of the range. Constraints 34] 36 are the typical linearizations of the products of a binary variable with a continuous one. Constraints 37- 42 define the domain of all variables in the formulation.

Remark 4 The range coverage being an increasing variable, and the initial range of coverage $c_{\text {init }}$ being determined by the value of parameter $Z_{s, N G}^{0}$ for each $s \in \mathcal{S}$, we can use set $C^{\prime}=\left\{c_{\text {init }}, \ldots, C\right\}$ instead of $C$, hence reducing the formulation size.

We show in the Appendix how this model can be extended to tackle frameworks where more than two generations co-exist.

\section{4 | STRENGTHENING AND UPPER BOUND}

We strengthen our formulation with several valid inequalities in Section 4.1 and provide in Section 4.2 a heuristic algorithm based on the properties of the problem where the offered subsidies and the coverage are fixed.

\section{1 | Valid inequalities}

Preliminary computational experiments on small instances showed that the solutions of linear relaxation present variables $z$ and $\delta$ fractional. Consequently, we propose several valid inequalities in this subsection to reinforce the model. The strength of these inequalities is assessed numerically in Section 5.2 
Proposition 5 Considering a time period $t \in \mathcal{T} \cup\{0\}$ and a site $s \in \mathcal{S}$, inequality

$$
z_{s, N G}^{t} \leq z_{s, N G}^{t+1}
$$

is valid for formulation $\mathcal{M}$.

Proof This result is implied by constraints [3] and 4].

Proposition 6 Considering a time period $t \in \mathcal{T}$ and a range of coverage $c \in C$, for all time periods $t^{\prime}$ posterior to $t$, inequality

$$
\sum_{\sigma \in \mathcal{K}} \sum_{c^{\prime}<c} \delta_{\sigma, c^{\prime}}^{t^{\prime}} \leq 1-\sum_{\sigma \in \mathcal{K}} \sum_{c^{\prime} \geq c} \delta_{\sigma, c^{\prime}}^{t}
$$

is valid for formulation $\mathcal{M}$.

Proof This set of constraints states that if at a time period $t \in \mathcal{T}$, the range of coverage is greater or equal to $c \in C$, then the range of coverage for posterior time periods cannot be smaller. As defined in Section 2 the NG sites coverage is indeed non-decreasing over the time horizon.

Proposition 7 Considering a time period $t \in \mathcal{T}$ and a site $s \in \mathcal{S}$, equality

$$
\sum_{c \in C} \sum_{\sigma \in \mathcal{K}} \pi_{\sigma, c, s, C G}^{t}=u_{s, C G}^{t-1}
$$

is valid for formulation $\mathcal{M}$.

Proof Following the Reformulation Linearization Techniques (see [21] for more details), we obtained these constraints by multiplying each constraint from set 31] by variables $u_{s, C G}^{t-1}$ for each $s \in \mathcal{S}$. The product obtained in the left member is then replaced by the corresponding linearization variable.

Proposition 8 Considering a time period $t \in \mathcal{T}$ and a range of coverage $c \in C$, inequality

$$
\left\lceil N_{S} L_{c}\right\rceil \sum_{\sigma \in \mathcal{K}} \delta_{\sigma, c}^{t} \leq \sum_{s \in \mathcal{S}} z_{s, N G}^{t}
$$

is valid for formulation $\mathcal{M}$.

Proof The network is in a range of coverage $c \in C$ only if technology $N G$ is deployed on at least $\left\lceil N_{S} L_{c}\right\rceil$ sites (remember than $N_{S}$ is the total number of sites and is constant over the time horizon).

Proposition 9 Let $\underline{\cup}_{s, N G}^{t}$ denote a lower bound on the number of NG subscribers on site $s$ at time period $t$. Considering a time period $t \in \mathcal{T}$ and $a$ site $s \in \mathcal{S}$, inequality

$$
\left\lceil\frac{D_{N G, N G}^{t} \underline{U}_{s, N G}^{t}}{C A P_{N G}}\right\rceil z_{s, N G}^{t} \leq m_{s, N G}^{t}
$$


is valid for formulation $\mathcal{M}$.

Proof If $N G$ technology is deployed on a site $s \in \mathcal{S}$ at a time period $t \in \mathcal{T}$, we know that $N G$ subscribers have to be served by $N G$ technology. By computing a lower bound on the quantity of $N G$ subscribers at this site and on this time period, we can hence compute a corresponding lower bound on the number of modules required for satisfying the capacity constraints 7 .

Proposition 10 Let $\bar{U}_{s, N G}^{t}$ denote an upper bound on the number of NG subscribers on site $s$ at time period $t$. Considering a time period $t \in \mathcal{T}$ and a site $s \in \mathcal{S}$, every optimal solution of the MMPP satisfies the following inequality:

$$
m_{s, N G}^{t} \leq \max \left(M_{s, N G}^{0},\left\lceil\frac{D_{N G, N G}^{t} \bar{U}_{s, N G}^{t}}{C A P_{N G}}\right\rceil\right) z_{s, N G}^{t} .
$$

Proof If $N G$ technology is not deployed on a site $s \in \mathcal{S}$ at a time period $t$, the number of modules for this technology on this site at this time period is 0 . If $N G$ technology is deployed on a site $s \in \mathcal{S}$ at a time period $t$, we know that only $N G$ subscribers on this site can be served by $N G$ technology. By computing an upper bound of the quantity of $N G$ subscribers on this site at this time period, we can hence compute a corresponding upper bound of the number of modules needed to satisfy the capacity constraints 7]. Installing more than this bound costs $C A_{N G}$ by additional module without any impact of the feasibility, and such a solution will hence be eliminated by the objective function minimization.

Remark 11 Values for bounds $\underline{U}_{s, N G}^{t}$ and $\bar{U}_{s, N G}^{t}$ are computed with the following recursive formulas:

$$
\begin{array}{ll}
\underline{U}_{s, N G}^{0}=\bar{U}_{s, N G}^{0}=U_{s, N G}^{0} & \forall t \in \mathcal{T} \\
\underline{U}_{s, N G}^{t}=\underline{U}_{s, N G}^{t-1}+N_{N G}^{t} U T O T_{s}^{t-1}+\left(U T O T_{s}^{t-1}-\underline{U}_{s, N G}^{t-1}\right) \min _{\sigma \in \mathcal{K}} f_{\sigma, c_{i n i t}} & \\
\bar{U}_{s, N G}^{1}=U_{s, N G}^{0}+N_{N G}^{t} U T O T_{s}^{0}+U_{s, C G}^{0} \max _{\sigma \in \mathcal{K}} f_{\sigma, c_{i n i t}} & \forall t \in\{2, \ldots, \bar{t}\} \\
\bar{U}_{s, N G}^{t}=\bar{U}_{s, N G}^{t-1}+N_{N G}^{t} U T O T_{s}^{t-1}+\left(U T O T_{s}^{t-1}-\bar{U}_{s, N G}^{t-1}\right) \max _{\sigma \in \mathcal{K}} f_{\sigma, C} &
\end{array}
$$

\subsection{Heuristic approach: fixing the upgrade reaction values}

In this section, we discuss a heuristic algorithm that can also help to solve the MMPP. The main difficulties of formulation $\mathcal{M}^{N L}$ come from the non-linear constraints 9], 10 and [14]. Hence, a simple heuristic approach to the problem would be to fix variables $\sigma^{t}$ (the amount of the subsidy offered to $C G$ subscribers) and $c^{t}$ (the coverage range) to specific values $\tilde{\sigma}^{t} \in C$ and $\tilde{c}^{t} \in \mathcal{K}$ for each period $t \in \mathcal{T}$ and solve the resulting problem optimally. Note that $\tilde{c}^{1}$ is already fixed to the initial coverage $c_{\text {init }}$. 
Let us now denote the problem where $\sigma^{t}=\tilde{\sigma}^{t}$ and $c^{t}=\tilde{c}^{t}$ as the $\operatorname{MMPP}(\tilde{\sigma}, \tilde{c})$. Applying constraints 9 recursively over the time horizon, we obtain that the numbers of CG subscribers is now a constant equal to

$$
U_{s, C G}^{t}=\left(1-f_{\tilde{\sigma}^{t}, \tilde{c}^{t}}\right) U_{s, C G}^{t-1}+N_{C G}^{t} U T O T_{s}^{t-1} \quad \forall t \in \mathcal{T}, \forall s \in \mathcal{S}
$$

By using parameter UTOT ${ }_{s}^{t}$, we hence have that the number of NG subscribers is now a constant equal to

$$
U_{s, N G}^{t}=U T O T_{s}^{t}-U_{s, C G}^{t} \quad \forall t \in \mathcal{T}, \forall s \in \mathcal{S}
$$

We can also compute the amount of money spent in subsidies offered to the subscribers, which is equal to the constant

$$
\text { upgradecost }=\sum_{t \in \mathcal{T}} \tilde{\sigma}^{t} f_{\tilde{\sigma}^{t}} U^{t} U_{s, C G}^{t-1} .
$$

We are now able to reformulate capacity and load-balancing constraints as a set of constraint depending only on the fixed constants $U_{s, C G}^{t}$ and $U_{s, N G}^{t}$ and of variables $m$ and $z$. Consequently, the $\operatorname{MMPP}(\tilde{\sigma}, \tilde{c})$ can be formulated as follows

$$
\begin{aligned}
& \min \text { upgradecost }+\sum_{s \in \mathcal{S}} \sum_{g \in \mathcal{G}} C M_{g}\left(m_{s, g}^{\bar{t}}-M_{s, g}^{0}\right) \\
& +\sum_{s \in \mathcal{S}} C A_{N G}\left(z_{s, N G}^{\bar{t}}-Z_{s, N G}^{0}\right)
\end{aligned}
$$

$$
\begin{array}{ll}
\text { s.t. } m_{s, C G}^{t} \leq \bar{M}_{C G} & \forall s \in \mathcal{S}, \forall t \in \mathcal{T}, \\
m_{s, N G}^{t} \leq \bar{M}_{N G} z_{s, N G}^{t} & \forall s \in \mathcal{S} \forall t \in \mathcal{T}, \\
m_{s, g}^{t-1} \leq m_{s, g}^{t} & \forall s \in \mathcal{S}, \forall t \in \mathcal{T}, \\
D_{C G}^{t} U_{s, C G}^{t}+D_{N G, C G}^{t} U_{s, N G}^{t}\left(1-z_{s, N G}^{t}\right) \leq C A P_{C G} m_{s, C G}^{t} & \forall s \in \mathcal{S}, \forall t \in \mathcal{T}, \\
D_{N G, N G}^{t} U_{s, N G}^{t} z_{s, N G}^{t} \leq C A P_{N G} m_{s, N G}^{t} & \forall s \in \mathcal{S}, \forall t \in \mathcal{T}, \\
\sum_{s \in \mathcal{S}} U_{s, N G}^{\bar{t}} z_{s, N G}^{\bar{t}} \geq \underline{Q} \underline{\underline{S}} \sum_{s \in \mathcal{S}} U_{s}^{T O T}, & \\
\sum_{s \in \mathcal{S}} z_{s, N G}^{\bar{t}} \geq \bar{\alpha} & \\
\sum_{s \in \mathcal{S}} z_{s, N G}^{t-1} \geq\left\lceil L_{\tilde{c}^{t}} N_{S}\right\rceil & \\
\sum_{s \in \mathcal{S}} z_{s, N G}^{t-1} \leq\left\lfloor U_{\tilde{c}^{t}} N_{S}\right\rfloor & \forall t \in \mathcal{T}, \\
m_{s, g}^{0}=M_{s, g}^{0} & \forall t \in \mathcal{T},
\end{array}
$$




$$
\begin{array}{ll}
z_{s, N G}^{0}=Z_{s, N G}^{0} & \forall s \in \mathcal{S}, \\
m_{s, g}^{t} \in \mathbb{N} & \forall s \in \mathcal{S}, \forall t \in \mathcal{T} \cup\{0\}, \forall g \in \mathcal{G}, \\
z_{s, N G}^{t} \in\{0,1\} & \forall s \in \mathcal{S}, \forall t \in \mathcal{T} \cup\{0\} .
\end{array}
$$

The objective [1] can be reformulated into 52. where the term standing for subsidies cost is now a constant. Constraints 56-57 ensure the load-balancing rules (seen from a network point of view rather than from a subscriber point of view). Constraints 56 ensure that CG technology has to handle the consumption of only $C G$ subscribers when $N G$ technology is installed and of both $C G$ and $N G$ subscribers when $N G$ technology is not installed. Constraints [57] ensure that when installed, NG technology has to handle the consumption of $N G$ subscribers. The threshold constraint can be reformulated as 58] since $N G$ subscribers are served by $N G$ if and only if $N G$ is installed. Constraints [59-61] are obtained by applying the definition of $\alpha$.

Remark 12 This formulation for the $\operatorname{MMPP}(\tilde{\sigma}, \tilde{c})$ still holds when smoothing constraints 25] are added.

For the remainder of the section, we will focus on simplified formulations for the MMPP $(\tilde{\sigma}, \tilde{c})$. First, we remove time dependency on variables $m$ (replacing $m_{s, g}^{t}$ by $m_{s, g}$ the number of modules installed at the end of the time horizon for each site $s$ and generation $g$ ), which means that smoothing constraints 25] cannot be added to the formulations provided (see Remark 2]. Moreover, let us recall that $D_{g, o}^{t-1} \leq D_{g, o}^{t}$ for each $g, o \in \mathcal{G}$ and for each $t \in \mathcal{T}$. Notice that for each $t \in \mathcal{T}$ and each site $s \in \mathcal{S}, U_{s, N G}^{t-1} \leq U_{s, N G}^{t}$. Consequently, the above formulation can be simplified significantly. Namely, one readily verifies that constraints [56- 57] are dominated by the following constraints:

$$
\begin{array}{ll}
D_{C G}^{t} U_{s, C G}^{t}+D_{N G, C G}^{t} U_{s, N G}^{t}\left(1-z_{s, N G}^{t}\right) \leq C A P_{C G} m_{s, C G} & \forall s \in \mathcal{S}, \forall t \in \mathcal{T}, \\
D_{N G}^{\bar{t}} U_{s, N G}^{\bar{t}} z_{s, N G}^{\bar{t}} \leq C A P_{N G} m_{s, N G} & \forall s \in \mathcal{S} .
\end{array}
$$

We observe that we can use these constraints to compute a closed form for the optimal value taken by variables $m_{s, g}$ which depends on the values taken by variables $z_{s, N G}^{t}$. We know indeed that for each site $s \in \mathcal{S}$ the number of modules installed at the end of the time horizon is:

- for $C G$ technology:

- if $N G$ is already installed $\left(Z_{s, N G}^{0}=1\right): \tilde{m}_{s, C G}^{A I}=\max \left(\left[\frac{\max _{i \in \mathcal{T}} D_{C G}^{i} U_{s, C G}^{i}}{C A P_{C G}}\right], M_{s, C G}^{0}\right)$ (only $C G$ subscribers are served by $C G$ technology),

- if $N G$ is not installed over the time horizon $\left(z_{s, N G}^{\bar{t}}=0\right)$ :

$$
\tilde{m}_{s, C G}^{N I}=\max \left(\left[\frac{\max _{i \in \mathcal{T}} D_{C G}^{i} U_{C G, s}^{i}+D_{N G, C G}^{i} U_{N G, s}^{i}}{C A P_{C G}}\right\rceil, M_{s, C G}^{0}\right)
$$

(all subscribers are served by CG technology), 
- if $N G$ is installed at time period $t \in \mathcal{T}\left(z_{s, N G}^{t}-z_{s, N G}^{t-1}=1\right)$ :

$$
\tilde{m}_{s, C G}^{t}=\max \left(\left[\frac{\max _{i<t} D_{C G}^{i} U_{C G, s}^{i}+D_{N G, C G}^{i} U_{N G, s}^{i}}{C A P_{C G}}\right\rceil,\left[\frac{\max _{i \geq t} D_{C G}^{i} U_{s, C G}^{i}}{C A P_{C G}}\right\rceil, M_{s, C G}^{0}\right),
$$

- for $N G$ technology when it is installed $\left(z_{s, N G}^{\bar{t}}=1\right): \tilde{m}_{s, N G}=\max \left(\left[\frac{D_{N G, N G}^{\bar{t}} U_{s, N G}^{\bar{t}}}{C_{N P S}}\right], M_{s, N G}^{0}\right)$.

Note that if, on a site $s \in \mathcal{S}, \tilde{m}_{s, C G}^{A I}>\bar{M}_{C G}$ then $z_{s, N G}^{\bar{t}}=1$ and if $\tilde{m}_{s, N G}>\bar{M}_{N G}$ then $z_{s, N G}^{\bar{t}}=0$. If both happen, the instance is unfeasible.

Consequently, we can provide a formulation for the $\operatorname{MMPP}(\tilde{\sigma}, \tilde{c})$ using only binary variables $z$.

$$
\begin{aligned}
\min & \sum_{s \in \mathcal{S}} \text { upgradecost }+C A_{N G}\left(z_{s, N G}^{\bar{t}}-Z_{s, N G}^{0}\right) \\
& +\left(\tilde{m}_{s, N G}-M_{s, N G}^{0}\right) C M_{N G} z_{s, N G}^{\bar{t}}+\left(\tilde{m}_{s, C G}^{N I}-M_{s, C G}^{0}\right) C M_{C G}\left(1-z_{s, N G}^{\bar{t}}\right) \\
& +\sum_{t \in \mathcal{T}}\left(\tilde{m}_{s, C G}^{t}-M_{s, C G}^{0}\right) C M_{C G}\left(z_{s, N G}^{t}-z_{s, N G}^{t-1}\right)+\left(\tilde{m}_{s, C G}^{A I}-M_{s, C G}^{0}\right) C M_{C G} Z_{s, N G}^{0}
\end{aligned}
$$

$$
\begin{array}{ll}
\text { s.t. } \sum_{s \in \mathcal{S}} U_{s, N G}^{\bar{t}} z_{s, N G}^{\bar{t}} \geq \underline{Q O E} \sum_{s \in \mathcal{S}} U \operatorname{UTOT}_{s}^{\bar{t}}, & \\
z_{s, N G}^{t-1} \leq z_{s, N G}^{t} & \forall t \in \mathcal{T}, \\
\sum_{s \in \mathcal{S}} z_{s, N G}^{\bar{t}} \geq \bar{\alpha}, & \\
\sum_{s \in \mathcal{S}} z_{s, N G}^{t-1} \geq\left\lceil L_{\tilde{c}^{t}} N_{S}\right\rceil & \forall t \in \mathcal{T}, \\
\sum_{s \in \mathcal{S}} z_{s, N G}^{t-1} \leq\left\lfloor U_{\tilde{c}^{t}} N_{S}\right\rfloor & \forall t \in \mathcal{T}, \\
z_{s, N G}^{0}=Z_{s, N G}^{0} & \forall s \in \mathcal{S}, \\
z_{s, N G}^{t} \in\{0,1\} & \forall s \in \mathcal{S}, \forall t \in \mathcal{T} .
\end{array}
$$

We define by $\mathcal{S}_{C G} \subset \mathcal{S}$ the subset of the sites where $N G$ is not installed the beginning of the time horizon. We also remove from set $\mathcal{S}_{C G}$ the sites for which we already know if we will install $N G$ technology or not due to unfeasibilities. We notice that we only have to solve the problem on sites of set $\mathcal{S}_{C G}$.

Our heuristic algorithm cannot afford to enumerate for each time period $t \in \mathcal{T}$ all possible values of $\left(\tilde{\sigma}^{t}, \tilde{c}^{t}\right) \in \mathcal{K} \times C$ since that would result in solving $(K \times C)^{\bar{t}}$ problems. This approach would actually solve the MMPP exactly. Instead, we propose to test a restricted subset $\mathcal{W} \subset(\mathcal{K} \times C)^{\bar{t}}$ of different couples of values for each time period, see Algorithm 1

As explained next, for tractability reasons we assume that

$$
\tilde{c}=\left(c_{i n i t}, C, \ldots, C\right) \text { for each }(\tilde{\sigma}, \tilde{c}) \in \mathcal{W} \text {. }
$$




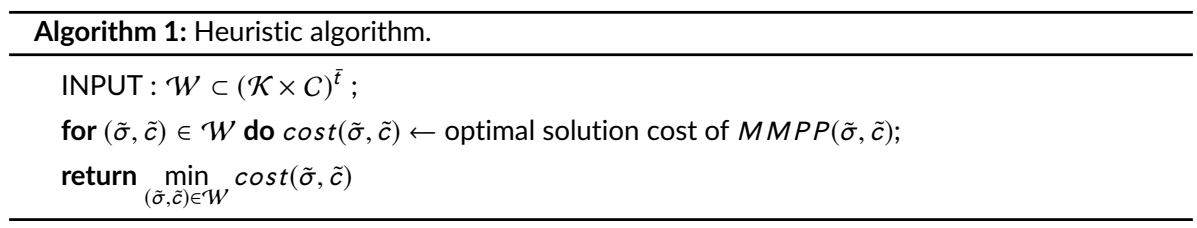

Assumption 76 implies that all network investments are performed in the first time period (see objective (68). Hence, we can replace variables $z_{s, N G}^{t}$ for each $t \in \mathcal{T}$ by variables $z_{s, N G}^{\bar{t}}$.

Remark 13 Assumption 76 cannot be made on the optimal solution of the MMPP. Let us consider an instance with one single site with 100 subscribers (total number of subscribers constant over the time horizon), initially all $C G$ subscribers. NG technology is not installed at the beginning of the time horizon. Parameter $Q O E$ is set to 60\%. Two subsidy amounts ( 0 and $100 €$ ) and two coverage ranges (high if $N G$ has been installed and low otherwise) are considered with $f_{0, \text { low }}=f_{0, \text { high }}=0, f_{100, \text { low }}=40 \%$ and $f_{100, \text { high }}=70 \%$. Three $3 \mathrm{G}$ modules are already installed, enough for handling all the traffic from the 100 subscribers on $3 G$ networks over the time horizon. One antenna NG has to be installed for a cost of $C A_{N G}$, and one module $N G$ has to be installed every $65 N G$ subscribers for a price of $C M_{N G}$. The optimal solution performs two subsidies in coverage low for a total cost of $C 1=C A_{N G}+C M_{N G}+6.4 k €$. Installing at the first period would impose to perform at least one subsidy in coverage high and would hence require higher network and subsidies costs: the total cost would be $C 2=C A_{N G}+2 C M_{N G}+7 k €$.

All modules costs for sites of set $\mathcal{S} \backslash \mathcal{S}_{C G}$ are labeled into constant netcost. Constant $N_{\text {inst }}$ labelled the number of sites where we know $N G$ is installed at the end of the time horizon. For each site $s \in \mathcal{S}_{C G}, C 1_{s}=C A_{N G}+\tilde{m}_{s, N G} C M_{N G}$ denotes the cost implied by deciding to install $N G$ technology and $C 2_{s}=\left(\tilde{m}_{s, C G}^{N I}-\tilde{m}_{s, C G}^{A I}\right) C M_{C G}$ the cost implied by deciding not to. Therefore, the $\operatorname{MMPP}(\tilde{\sigma}, \tilde{c})$ can be further reformulated as the following bidimensional knapsack problem:

$$
\begin{aligned}
& \min \sum_{s \in \mathcal{S}_{C G}} C 1_{s} z_{s, N G}^{\bar{t}}+C 2_{s}\left(1-z_{s, N G}^{\bar{t}}\right)+\text { upgradecost }+ \text { netcost } \\
& \text { st } \sum_{s \in \mathcal{S}_{C G}} U_{s, N G}^{\bar{t}} z_{s, N G}^{\bar{t}} \geq \underline{Q} \underline{\underline{Q}} \sum_{s \in \mathcal{S}} U_{s}^{T O T}-\sum_{s \in \mathcal{S} \backslash \mathcal{S}_{C G}} U_{s, N G}^{\bar{t}}, \\
& \quad \sum_{s \in \mathcal{S}_{C G}} z_{s, N G}^{\bar{t}} \geq \max \left(\bar{\alpha}, L_{C} N_{S}\right)-N_{i n s t}, \\
& z_{s, N G}^{\bar{t}} \in\{0,1\} \quad \forall s \in \mathcal{S}_{C G} .
\end{aligned}
$$

Proposition 14 The $\operatorname{MMPP}(\tilde{\sigma}, \tilde{c})$ can be solved in $O\left(N S+\left|\mathcal{S}_{C G}\right|^{2}\left(C A_{N G}+\bar{M}_{N G} C M_{N G}\right)\right)$ when $\tilde{c}^{t}=$ $C, \forall t \in\{2, \ldots, \bar{t}\}$.

Proof From the arguments given above, this result follows from the fact that the problem becomes a knapsack problem which can be solved by a dynamic programming algorithm. 


\section{5 | CASE STUDY FOR 3G AND 4G}

The purpose of this case study is two-fold. First, we assess the scalability of the exact MILP model, the impact of the proposed valid inequalities and the quality of our heuristic algorithm. Second, we observe the characteristics of the solutions in terms of costs, considering several businesses scenarios, including when the investment expenses are smoothed along the time horizon.

\section{1 | Instances and platform}

Numerical tests are performed on instances of French telecommunication operator Orange in the French areas of Brittany and Pays de la Loire representing a case study with two network generations: 3G and 4G. The full area contains 1075 sites: 700 equipped only with 3G technology and 375 equipped with both technologies. We create a set of smaller instances, out of this large instance, in order to have different scenarios characterized by the number of sites and the $4 \mathrm{G}$ initial coverage: rural scenarios where the $4 \mathrm{G}$ technology is initially deployed on $17 \%$ of the sites, suburban scenarios where this proportion is equal to $34 \%$ and urban scenarios where it is equal to $68 \%$.

The real data include the number of modules and subscribers for each site. There are no new incomers in theses instances, and the only evolution in the number of subscribers is hence due to subsidy. Others parameter values of this case study are realistic values taken from telecommunication equipment sellers. Each site can carry a maximum of four $3 \mathrm{G}$ modules (carrier) of $5 \mathrm{MHZ}$ with a capacity of $3 \mathrm{Mbps}$ and a cost of $3 \mathrm{k} €$ each and a maximum of five $4 \mathrm{G}$ modules of $10 \mathrm{MHZ}$ (bandwidth) with a capacity of $25 \mathrm{Mbps}$ and a cost of $16 \mathrm{k} €$ each. The cost for adding the $4 \mathrm{G}$ technology on a site is $75 \mathrm{k} €$. The subscribers monthly demands are forecasted: $D_{3 G}=D_{4 G, 3 G}$ increase from 1 to $2 \mathrm{~Gb}$ per month; while $D_{4 G, 4 G}$ increases from 2 to $5 \mathrm{~Gb}$ per month. As for subsidies, we considered 10 values for the of subsidy offered, $\sigma \in\{0,100,150,200,250,300,350,400,450,500\} €$. We also rely on four levels of $4 \mathrm{G}$ sites coverage: low, medium low, medium high and high respectively refer to ranges (in\%) [0, 25[, [25, 50[, [50, 75[ and [75, 100]. The curves from Figure 3 are hence discretized into the table of values shown in Table 3 which constitutes our reference upgrade function. Two other upgrades functions are also considered for adapting to very technology-reluctant

\begin{tabular}{|c|c|c|c|c|c|c|c|c|c|c|}
\hline Coverage level \Subsidies (in €) & 0 & 100 & 150 & 200 & 250 & 300 & 350 & 400 & 450 & 500 \\
\hline low & 0 & 0 & 0 & 0 & 0 & 0 & 0 & 0 & 0 & 0 \\
\hline medium low & 0.5 & 5 & 12 & 21 & 30 & 40 & 42 & 43 & 44 & 45 \\
\hline medium high & 5 & 10 & 20 & 30 & 40 & 50 & 60 & 62 & 64 & 65 \\
\hline high & 10 & 20 & 33 & 45 & 58 & 70 & 80 & 83 & 87 & 90 \\
\hline
\end{tabular}

TAB LE 3 Reaction of the subscribers (in \%) on reference markets for given subsidies and coverage levels.

markets and technology-friendly markets. We ask for final target objectives of $Q \circ E=80 \%$ for the quality of experience and $\underline{\alpha}=70 \%$ for the $4 \mathrm{G}$ sites coverage. We optimize on the typical time 
horizon of five years discretized in five time periods of one year. The unit of the objective value is $k €$.

The computations have been made on a server of 32 processors Intel Xeon of CPU 5110 clocked at $1.6 \mathrm{GHz}$ each. The code has been written in Julia 1.1.0, and the solver used is CPLEX 12.8 (default branch-and-bound algorithm). The time limit for MILP solving is set to 1800 seconds for Sections 5.2 and 5.3 We have observed in our experiments that removing the dependency of variables $m$ on time period $t$ does not help improving the solution found nor the gap.

\section{2 | Results for algorithmic tests}

Our objectives in this section are to assess the scalability of our formulation (including the impact of the proposed valid inequalities) and to test its sensitivity to the type of areas. Hence we focus on twelve instances considering four sizes between 50 and 200 sites and the three types of areas mentioned above. The upgrade function used here is the reference one (see Table 3. Instances features are displayed in Tables 4 and 6 column " $N$ " standing for the number of sites and column "density" standing for the density scenario (rural $R$, suburban $S$ or urban $U$ ). The solutions of the linear relaxation and of the MILP are computed with and without the valid inequalities from Section 4.1. More precisely, we test formulations $(\mathcal{M}),(\mathcal{M}+$ each family of valid inequality) and $(\mathcal{M}+$ all families of valid inequalities). The obtained root gap for each tested formulation is displayed in Table 4 The root gap is given in percentage, and is obtained by dividing the difference between the best solution found and the value of the continuous relaxation, by the value of the best solution found. The best root gap among the formulations with a single valid inequality is in bold. For each formulation, the value of the best solution found by CPLEX within the time limit is displayed in Table 5 We label the value with the character ${ }^{(*)}$ if the branch-and-bound procedure converged. The best solution found is in bold. The corresponding final gap is displayed in Table 6 The final gap is given in percentage, and is obtained by dividing the difference between the best solution and the best lower bound found, by the value of the best solution. The best value for the final gap is in bold and the second best is in italic.

First, we provide insight on the relative efficiency of each family of valid inequalities. We observe, on Table 4 tighter relaxations when using the valid inequalities. More precisely, a significant improvement on the relaxation is enabled by the RLT set of inequalities 45. Improvements on the root gaps can also be seen in urban instances by adding the sets 43 or 48 . Indeed, when the new technology is already deployed on most sites, the non-decreasing of $z$ reduces the search space. For this reason, these instances are the ones for which adding all inequalities rather than only inequalities 45 significantly improves the root gap. Moreover, we observe that inequalities 45] also have the best impact among the different valid inequalities for reducing the final gap (see Table 6. The solution found with inequalities 45 is always the best one found, as it can be observed in Table 5 but using the inequalities all together enables us to find the same solutions, with (for most instances) a slightly lower final gap.

For the following tests and observations, we will hence focus on the formulation including all families of valid inequalities. First, referring to scalability, we observe that the branch-and-bound 
TAB LE 4 Root gap values for 12 instances (4 sizes, 3 densities) tested with each family of valid inequalities.

\begin{tabular}{|c|c|c|c|c|c|c|c|c|c|}
\hline \multicolumn{2}{|c|}{ Instance } & \multicolumn{8}{|c|}{ Root gap } \\
\hline$N_{S}$ & density & $\mathcal{M}$ & +43 & +44 & +45 & + 46) & +47 & +48 & +43 - 48, \\
\hline \multirow[t]{3}{*}{50} & $R$ & 25 & 25 & 25 & 19 & 25 & 25 & 25 & 19 \\
\hline & S & 32 & 32 & 32 & 24 & 32 & 32 & 32 & 24 \\
\hline & U & 61 & 58 & 61 & 45 & 61 & 61 & 55 & 27 \\
\hline \multirow[t]{3}{*}{100} & $R$ & 26 & 26 & 26 & 20 & 26 & 26 & 26 & 20 \\
\hline & S & 31 & 32 & 31 & 24 & 31 & 31 & 31 & 23 \\
\hline & u & 62 & 59 & 62 & 47 & 62 & 62 & 57 & 28 \\
\hline \multirow[t]{3}{*}{150} & $\mathrm{R}$ & 28 & 25 & 25 & 20 & 26 & 25 & 25 & 20 \\
\hline & $S$ & 38 & 31 & 31 & 24 & 31 & 32 & 31 & 24 \\
\hline & U & 63 & 59 & 62 & 47 & 62 & 62 & 57 & 29 \\
\hline \multirow[t]{3}{*}{200} & $\mathrm{R}$ & 28 & 25 & 24 & 18 & 24 & 24 & 25 & 18 \\
\hline & S & 36 & 31 & 31 & 23 & 30 & 32 & 30 & 23 \\
\hline & u & 63 & 59 & 62 & 46 & 62 & 62 & 56 & 29 \\
\hline
\end{tabular}

TAB LE 5 Best solution for 12 instances (4 sizes, 3 densities) tested with each family of valid inequalities.

\begin{tabular}{|c|c|c|c|c|c|c|c|c|c|}
\hline \multicolumn{2}{|c|}{ Instance } & \multicolumn{8}{|c|}{ Best solution found by CPLEX } \\
\hline$N_{S}$ & density & $\mathcal{M}$ & +43 & +44 & +45 & +46 & +47 & +48 & $+43-48$ \\
\hline \multirow[t]{3}{*}{50} & $R$ & 4173 & $4103^{*}$ & $4103^{*}$ & $4103^{*}$ & $4103^{*}$ & $4103^{*}$ & $4103^{*}$ & $4103^{*}$ \\
\hline & $\mathrm{s}$ & 3458 & $3458^{*}$ & $3458^{*}$ & $3458^{*}$ & $3458^{*}$ & $3458^{*}$ & $3458^{*}$ & $3458^{*}$ \\
\hline & u & $2021^{*}$ & $2021^{*}$ & $2021^{*}$ & $2021^{*}$ & $2021^{*}$ & $2021^{*}$ & $2021^{*}$ & $2021^{*}$ \\
\hline \multirow[t]{3}{*}{100} & $\mathrm{R}$ & 8347 & 8347 & 8347 & 8347 & 8401 & 8347 & 8347 & 8347 \\
\hline & $\mathrm{s}$ & 7036 & 6902 & 6861 & 6861 & 6861 & 6861 & 6861 & 6861 \\
\hline & $U$ & 3864 & $3861^{*}$ & $3861^{*}$ & $3861^{*}$ & $3861^{*}$ & $3861^{*}$ & $3861^{*}$ & $3861^{*}$ \\
\hline \multirow[t]{3}{*}{150} & $\mathrm{R}$ & 12783 & 12308 & 12247 & 12242 & 12344 & 12247 & 12263 & 12242 \\
\hline & $\mathrm{s}$ & 11179 & 10049 & 10027 & 9990 & 10049 & 10146 & 10030 & 9990 \\
\hline & U & 5692 & $5522^{*}$ & $5522^{*}$ & $5522^{*}$ & $5522^{*}$ & $5522^{*}$ & $5522^{*}$ & $5522^{*}$ \\
\hline \multirow[t]{3}{*}{200} & $R$ & 17021 & 16309 & 16167 & 16036 & 16127 & 16036 & 16318 & 16036 \\
\hline & $\mathrm{s}$ & 14266 & 13305 & 13305 & 13094 & 13094 & 13567 & 13221 & 13094 \\
\hline & $\mathrm{u}$ & 7828 & 7616 & 7616 & 7616 & 7619 & 7616 & 7619 & 7616 \\
\hline
\end{tabular}


procedure converges to optimality for the three instances of 50 sites and the urban instances of 100 and 150 sites (and nearly converges for the urban instance of 200 sites). Besides, the final gap remain under $4 \%$ for all instances. Second, we focus on the sensitivity to the type of area. We notice that the problem proves easier to solve in urban areas, which can be explained by the decisions on coverage extension needed to satisfy the strategic targets: having more sites already covered by the newest technology results indeed in fewer decisions to take. However, the relaxation is weaker (see Table 4] due to the shape of the subsidy function (beginning with an high coverage means higher reactions but also higher gaps between continuous reactions and discrete reactions). This enlightens that the problem practical difficulty is strongly correlated with the question of coverage extension. We notice that these resulting coverage extension investments also have a significant financial impact. For instance, the optimal solution for the rural instance of 50 sites is around two times more expensive than the optimal solution for the urban instance.

TAB LE 6 Final gaps for 12 instances (4 sizes, 3 densities) tested with each family of valid inequalities.

\begin{tabular}{|c|c|c|c|c|c|c|c|c|c|}
\hline \multicolumn{2}{|c|}{ Instance } & \multicolumn{8}{|c|}{ Final gap } \\
\hline$N_{S}$ & density & $\mathcal{M}$ & +43 & +44 & +45 & +46 & +47 & +48 & +43 \\
\hline \multirow[t]{3}{*}{50} & $\mathrm{R}$ & 6.00 & 0.00 & 0.00 & 0.00 & 0.00 & 0.00 & 0.00 & 0.00 \\
\hline & $\mathrm{S}$ & 5.97 & 0.00 & 0.00 & 0.00 & 0.00 & 0.00 & 0.00 & 0.00 \\
\hline & U & 0.00 & 0.00 & 0.00 & 0.00 & 0.00 & 0.00 & 0.00 & 0.00 \\
\hline \multirow[t]{3}{*}{100} & $\mathrm{R}$ & 10.62 & 4.52 & 3.13 & 3.59 & 6.33 & 1.90 & 6.46 & 1.14 \\
\hline & $\mathrm{S}$ & 14.92 & 4.81 & 3.17 & 2.55 & 3.60 & 3.67 & 4.37 & 2.50 \\
\hline & $U$ & 7.18 & 0.00 & 0.00 & 0.00 & 0.00 & 0.00 & 0.00 & 0.00 \\
\hline \multirow[t]{3}{*}{150} & $\mathrm{R}$ & 16.71 & 9.65 & 5.62 & 4.43 & 7.91 & 6.87 & 6.87 & 4.12 \\
\hline & $\mathrm{s}$ & 20.72 & 10.49 & 4.34 & 3.91 & 4.13 & 10.45 & 7.71 & 3.47 \\
\hline & $U$ & 7.22 & 0.00 & 0.00 & 0.00 & 0.00 & 0.00 & 0.00 & 0.00 \\
\hline \multirow[t]{3}{*}{200} & $\mathrm{R}$ & 16.73 & 10.53 & 7.99 & 3.59 & 10.55 & 8.84 & 9.80 & 2.48 \\
\hline & $\mathrm{s}$ & 18.85 & 10.06 & 12.22 & 2.77 & 11.03 & 13.59 & 12.28 & 3.06 \\
\hline & U & 7.73 & 1.83 & 1.01 & 0.49 & 1.96 & 1.86 & 2.27 & 0.07 \\
\hline
\end{tabular}

\section{3 | Results for business-oriented tests}

In this section, we assess the impact of several business-oriented scenarios from both algorithmic and financial perspectives:

- smoothing the costs over the time horizon,

- considering three upgrade functions: the reference upgrade used in the algorithmic tests, the 
technology-reluctant and technology-friendly upgrades.

The technology-reluctant and technology-friendly upgrades functions considered are given in Tables 7 and 8 respectively.

\begin{tabular}{|c|c|c|c|c|c|c|c|c|c|c|}
\hline Coverage level \Subsidies (in €) & 0 & 100 & 150 & 200 & 250 & 300 & 350 & 400 & 450 & 500 \\
\hline low & 0 & 0 & 0 & 0 & 0 & 0 & 0 & 0 & 0 & 0 \\
\hline medium low & 0 & 1 & 5 & 10 & 15 & 20 & 25 & 26 & 27 & 28 \\
\hline medium high & 1 & 5 & 10 & 20 & 30 & 35 & 40 & 45 & 50 & 50 \\
\hline high & 5 & 10 & 23 & 36 & 48 & 55 & 60 & 65 & 70 & 70 \\
\hline
\end{tabular}

TABLE 7 Reaction of the subscribers (in \%) on Technology-Reluctant markets for given subsidies and coverage levels.

\begin{tabular}{|c|c|c|c|c|c|c|c|c|c|c|}
\hline Coverage level \Subsidies (in €) & 0 & 100 & 150 & 200 & 250 & 300 & 350 & 400 & 450 & 500 \\
\hline low & 0 & 1 & 5 & 10 & 15 & 20 & 25 & 26 & 27 & 28 \\
\hline medium low & 1 & 5 & 10 & 20 & 30 & 35 & 40 & 45 & 50 & 50 \\
\hline medium high & 7 & 12 & 23 & 36 & 48 & 55 & 62 & 69 & 75 & 75 \\
\hline high & 20 & 30 & 40 & 55 & 70 & 80 & 87 & 92 & 95 & 95 \\
\hline
\end{tabular}

TABLE 8 Reaction of the subscribers (in \%) on Technology-Friendly markets for given subsidies and coverage levels.

For these purposes, and in order to have a sufficient expected number of optimal solutions (according to the algorithmic tests) for assessing financial aspects, we consider a set of 18 instances made of

- for the 50 sites instances: the three densities and the three types of market ( 9 instances)

- for the 100 to 200 sites instances: suburban density and the three types of markets (9 instances).

These 18 instances are presented under "Instances" in Table 9 Columns " $N_{S}$ " and "density" have the same meaning as in Section 5.2 The type of market is displayed in column "upgrade": "T.F markets","Ref. markets" and "T.R. markets" stand, respectively for technology-friendly, reference and technology-reluctant markets.

We want to assess on these instances the impact of cost smoothing, i-e when we add constraints 25. These constraints enforce all period expenses to lie between $(1-p)$ and $(1+p)$ times the quotient of the total expenses over the time horizon by the number of time periods, where $p$ is a parameter setting the maximal allowed budget fluctuation - set at $10 \%$ for the following tests.

Note that the valid inequalities still hold, except for inequalities [48, which is hence removed. Results for both formulations " $\mathcal{M}$ " and " $\mathcal{M}+$ cost equilibrium" are displayed in Table 9 For each 
formulation, the indicators provided are the best solution found in column "sol", the final gap in column "f-gap" and the root gap in column "r-gap". These indicators have the same definition as in Section 5.2 The last column "overcost" gives the resulting overcost (the relative gap in \% between the values of the solutions without and with the cost equilibrium set of constraints).

From a computational point of view, adding the cost equilibrium constraints hardens the problem. We see indeed in Table 9 that the proof of optimality is obtained only for the urban instances of 50 sites.

TAB LE 9 Best solution found, final gap and root gap for cost equilibrium and other upgrade functions.

\begin{tabular}{|c|c|c|c|c|c|c|c|c|c|}
\hline \multicolumn{3}{|c|}{ Instance } & \multicolumn{3}{|c|}{$\mathcal{M}$} & \multicolumn{3}{|c|}{$\mathcal{M}+$ cost equilibrium } & \multirow[t]{2}{*}{ overcost } \\
\hline$N_{S}$ & density & upgrade & sol & f-gap & r-gap & sol & f-gap & r-gap & \\
\hline \multirow[t]{9}{*}{50} & $\mathrm{R}$ & T.F. markets & 3622 & 0.00 & 23 & 4410 & 5.19 & 32 & 22 \\
\hline & & Ref. markets & 4103 & 0.00 & 19 & 4589 & 0.90 & 26 & 12 \\
\hline & & T.R. markets & 4417 & 0.00 & 19 & 4962 & 2.05 & 26 & 12 \\
\hline & $S$ & T.F. markets & 2890 & 0.00 & 30 & 3600 & 1.73 & 40 & 25 \\
\hline & & Ref. markets & 3458 & 0.00 & 23 & 3877 & 1.70 & 31 & 12 \\
\hline & & T.R. markets & 3847 & 0.00 & 23 & 4192 & 0.04 & 28 & 9 \\
\hline & $u$ & T.F. markets & 1264 & 0.00 & 43 & 1742 & 0.00 & 77 & 38 \\
\hline & & Ref. markets & 2021 & 0.00 & 27 & 2043 & 0.00 & 11 & 1 \\
\hline & & T.R. markets & 2443 & 0.00 & 22 & 2470 & 0.00 & 34 & 1 \\
\hline \multirow[t]{3}{*}{100} & $S$ & T.F. markets & 5729 & 0.00 & 29 & 7142 & 2.54 & 40 & 25 \\
\hline & & Ref. markets & 6861 & 0.25 & 23 & 7767 & 7.95 & 31 & 13 \\
\hline & & T.R. markets & 7625 & 2.83 & 22 & 8389 & 7.64 & 28 & 10 \\
\hline \multirow[t]{3}{*}{150} & $S$ & T.F. markets & 8459 & 1.71 & 30 & 13650 & 26.22 & 54 & 61 \\
\hline & & Ref. markets & 9990 & 0.98 & 23 & 11642 & 11.12 & 34 & 17 \\
\hline & & T.R. markets & 11150 & 3.59 & 23 & 12130 & 6.66 & 29 & 9 \\
\hline \multirow[t]{3}{*}{200} & $S$ & T.F. markets & 11075 & 3.27 & 28 & 17984 & 26.15 & 53 & 62 \\
\hline & & Ref. markets & 13094 & 3.06 & 23 & 35768 & 52.99 & 71 & 173 \\
\hline & & T.R. markets & 14517 & 3.43 & 22 & 15949 & 6.49 & 28 & 10 \\
\hline
\end{tabular}

From a financial point of view, we consider the 50 sites instances in order to analyze the characteristics of an optimal solution. We thus draw the features of the solution for the 50 sites suburban instance with the upgrade for the reference markets, when we do not require the cost to be smoothed, in Figure 4 The 4G sites coverage, the amount of subsidies given and the reaction of the 
subscribers are plotted. On the $4 \mathrm{G}$ sites coverage curve, we can notice that the $4 \mathrm{G}$ sites coverage at the end of the time horizon is $80 \%$ and that this value is not reached progressively throughout the time horizon. Indeed, the $4 \mathrm{G}$ sites coverage at the end of the first year is already nearly equal to this final value. This fast deployment is made to benefit from more upgrade thanks to coverage improvements (for instance the natural effect of coverage improvement can be observed at the second time period, over which the switch from coverage range medium low to range high enables the model not to offer any subsidy). This enlightens the financial interest for the operator in quickly having a network of good quality. However, it results also in large budget variations, with the first year costing more than four times the second most expensive year, and almost nothing spent over the second year, as we can see in Figure 5 These important variations do not match with the financial context of a telecommunication operator as investments should be distributed along the whole time horizon.

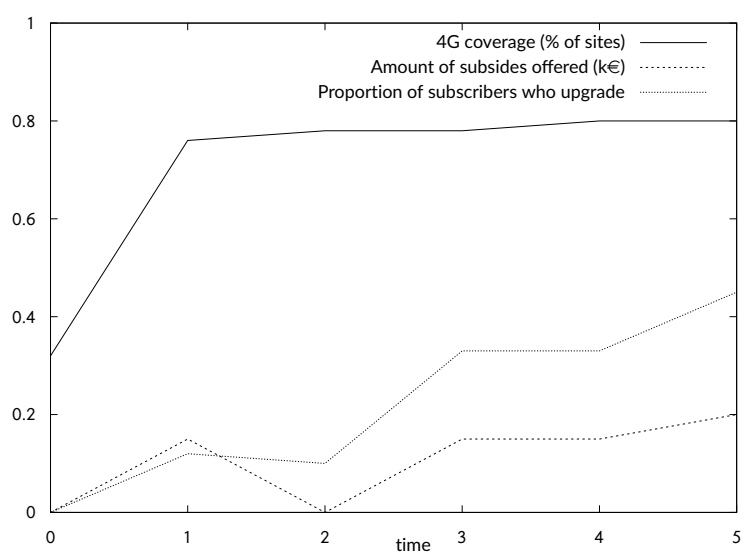

FIGURE 4 Evolution of the coverage, subsidies decisions and reactions over the time horizon (optimal solution of 50 sites suburban instance). 


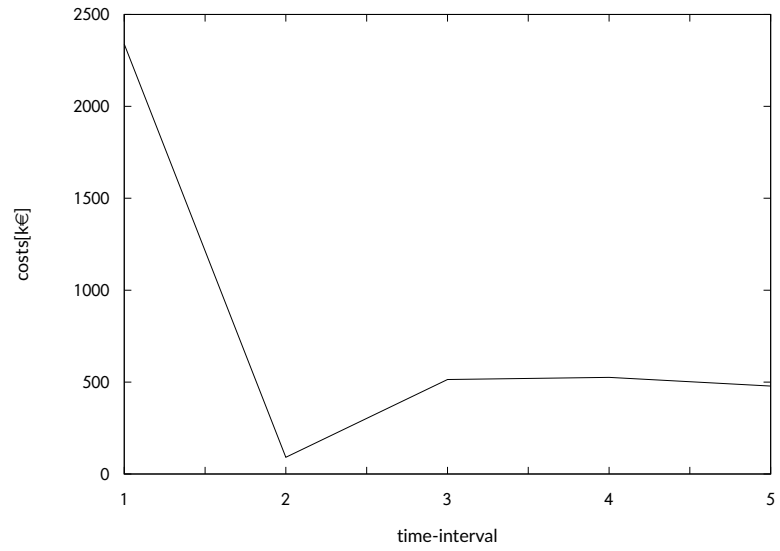

FIGURE 5 Evolution of the costs over the time horizon (optimal solution of 50 sites suburban instance).

We plot in Figure 6 the counterpart of Figure 4 when cost are required to be smoothed. We see in these curves that the range of coverage high is reached in four years in the solution with cost equilibrium instead of one without cost equilibrium (see Figure 4. The effect of the subsidies is hence considerably weakened, which can be observed on the reaction curve of Figure 6 This has an impact on the upgrade investments, which become higher (for instance $250 €$ per user instead of 200 are offered in the last year). Besides, in the second year, a subsidy of $150 €$ is offered while in the solution without imposing cost equilibrium the coverage improvements enabled the model not to offer any subsidy. These have important effects on the costs of the corresponding time periods, as can be observed in Figures 5 and 7 By comparing the previous instance with the other 50 sites instances for reference markets in rural and urban areas, we see the influence of the initial density on the overcost resulting from the cost smoothing. This effect can be seen in Table 9 column "overcost" and rows Ref.markets: the overcost is around $12 \%$ for rural and suburban instances while it is only $1 \%$ for urban ones. The needed investments for reaching the upper range of coverages are indeed lower when starting from higher initial 4G sites coverages, reducing gap between solutions with and without cost equilibrium. We hence are able to quantify the overcost to get business-fit solutions and see that this overcost is particularly reduced for urban instances.

Finally, with regards to the type of markets, we notice that there seems to be no algorithmic sensitivity to the choice of the upgrade function. In what concerns the financial sensitivity, we notice that, as expected, since more upgrade investments are needed, the cost is higher on reluctant markets. For instance, the cost for the suburban instance of 50 sites with the upgrade for reluctant markets is $33 \%$ more expensive than the instance with the upgrade for technology-friendly markets. 


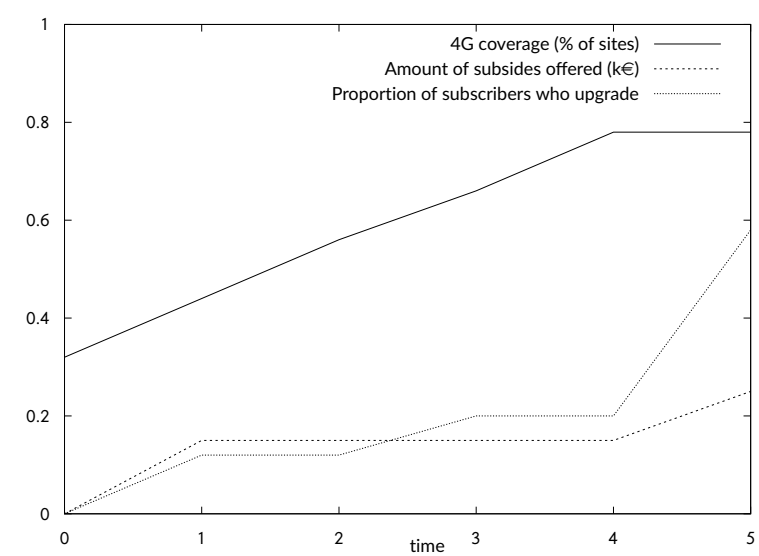

FIGURE 6 Evolution of the coverage, subsidies decisions and reactions over the time horizon (solution of 50 sites suburban instance) when cost equilibrium constraints are imposed.

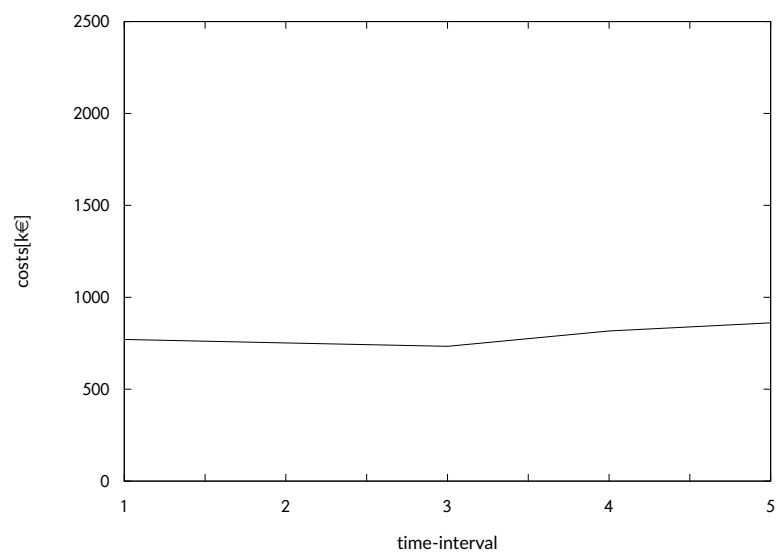

FIGURE 7 Evolution of the costs over the time horizon (solution of 50 sites suburban instance) when cost equilibrium constraints are imposed.

\subsection{Computational tests on large instances}

In this section, we assess our exact solution method on 10 instances corresponding to different French territorial divisions and compare it with the heuristic of Section 4.2. Two regions: Bretagne (divided into 4 departments: Finistère, Côtes d'Armor, Morbihan and lle et Vilaine) and part of Pays de la Loire (divided into 3 departments: Mayenne, Sarthe, Maine et Loire) are hence considered. As the planification is made for 5 years, the computational time is not what matters most for the operator, so we test larger time limits in order to see if it enables us to find better solutions and to 
reduce the gaps.

Instances are displayed in Table 10 The name of the territorial division (department/region), its number of sites and its initial 4G sites coverage in \% are respectively stored under "Ter. Div.", " $N_{\mathcal{S}}$ " and " $\alpha$ ". The best solution found and the final gap are labelled in the same way as above, and indicated for three different time limits: half-an hour, two hours and five hours.

We observe that, with a five-hour time limit, the final gap obtained is below $5 \%$ for 8 large real-life instances. However, the model struggles to find a good quality solution for the two largest instances.

TAB LE 10 Solution and final gap for large instances.

\begin{tabular}{|c|c|c||c|c||c|c||c|c|}
\hline \multicolumn{2}{|c||}{ Instance } & \multicolumn{2}{c||}{ MILP (half an hour) } & \multicolumn{2}{c||}{ MILP (two hours) } & \multicolumn{2}{|c|}{ MILP (five hours) } \\
\hline Ter. Div. & $N_{S}$ & $\alpha^{0}$ & sol & f-gap & sol & f-gap & sol & f-gap \\
\hline Finistère & 210 & 36 & 13885 & 7.00 & 13406 & 4.91 & 13406 & 4.61 \\
\hline Côtes d'Armor & 149 & 29 & 10420 & 3.38 & 10420 & 1.94 & 10420 & 1.48 \\
\hline Morbihan & 168 & 38 & 11178 & 4.08 & 11178 & 3.32 & 11178 & 2.75 \\
\hline Ile et Vilaine & 214 & 43 & 12400 & 3.76 & 12115 & 2.73 & 12115 & 2.15 \\
\hline Mayenne & 73 & 31 & 4879 & 1.62 & 4879 & 0.92 & 4879 & 0.50 \\
\hline Sarthe & 116 & 33 & 7729 & 3.23 & 7729 & 2.39 & 7728 & 1.64 \\
\hline Maine et Loire & 145 & 28 & 9877 & 4.68 & 9877 & 4.05 & 9877 & 3.65 \\
\hline Bretagne & 741 & 37 & $\infty$ & $\infty$ & 128109 & 100.00 & 128109 & 57.03 \\
\hline Pays de la Loire & 334 & 30 & $\infty$ & $\infty$ & 22470 & 4.26 & 22464 & 4.00 \\
\hline Full instance & 1075 & 35 & $\infty$ & $\infty$ & 169968 & 92.80 & 169968 & 92.80 \\
\hline
\end{tabular}

We hence assess the interest of the heuristic of Section 4.2 for finding feasible solutions. As mentioned previously, we will look for a solution where the range ("high") is reached over the first period and we enumerate the amount of subsidies $\tilde{\sigma} \in \mathcal{W}$ (ten possibilities if we do not restrict) at each time period (five) so as to solve each resulting problem $\operatorname{MMPP}(\tilde{\sigma}, \tilde{c})$ with the pseudo-polynomial model provided in Section 4.2. This means that we have to solve $10^{5} \mathrm{MMPP}(\tilde{\sigma}, \tilde{c})$ problems, which we cannot afford. In addition, we know that when the subsidies reaction is not sufficient to reach the threshold of $80 \%$ of subscribers being $4 G$ subscribers, the corresponding problem is unfeasible. We also observe that with 250 euros of subsidies at each period, we have less than $1 \%$ of the inital $3 \mathrm{G}$ subscribers remaining at the end of the time horizon, and that this situation is feasible for our instances. Hence, in our heuristic, we enumerate all $\tilde{\sigma} \in\{0,100,150,200,250\}$ and we solve the problem only if the reaction is sufficient to reach the threshold. This give at most $5^{5}=3125$ $\operatorname{MMPP}(\tilde{\sigma}, \tilde{c})$ problems to solve.

In a second step, the solution found by the heuristic is used as initial solution (MIPstart) for the solver. The time limit given to the solver is 7200 seconds minus the time of the heuristic in order to compare with the MIP solving in 7200 seconds without initial solution provided. 
Results are presented in Table 11 The column "heuristic" stands for the algorithm described above, the column "MILP" for the MILP without initial solution provided and the column "MIPstart" for the MILP with the heuristic solution provided as MIPstart. The column "gapMILP" reports the gap between the heuristic value and the MILP solution value.

TAB LE 11 Solution and final gap for large instances.

\begin{tabular}{|c|c|c|c|c||c||c|c||c|c|}
\hline \multicolumn{2}{|c|}{ Instance } & \multicolumn{2}{c||}{ heuristic } & gapMIP & \multicolumn{3}{c|}{ MILP } & \multicolumn{2}{c|}{ MIPstart } \\
\hline Ter. Div. & $N_{S}$ & $\alpha^{0}$ & sol & time & & sol & f-gap & sol & f-gap \\
\hline Finistère & 210 & 36 & 13406 & 505 & 0 & 13406 & 4.91 & 13406 & 3.14 \\
\hline Côtes d'Armor & 149 & 29 & 10420 & 617 & 0 & 10420 & 1.94 & 10420 & 1.64 \\
\hline Morbihan & 168 & 38 & 11178 & 551 & 0 & 11178 & 3.32 & 11178 & 2.06 \\
\hline Ile et Vilaine & 214 & 43 & 12115 & 776 & 0 & 12115 & 2.73 & 12115 & 2.32 \\
\hline Mayenne & 73 & 31 & 4879 & 127 & 0 & 4879 & 0.92 & 4879 & 0.00 \\
\hline Sarthe & 116 & 33 & 7729 & 186 & 0 & 7729 & 2.38 & 7229 & 0.00 \\
\hline Maine et Loire & 145 & 28 & 9877 & 221 & 0 & 9877 & 4.06 & 9877 & 0.72 \\
\hline Bretagne & 741 & 37 & 47106 & 3197 & -63.41 & 128109 & 100.00 & 47106 & 3.51 \\
\hline Pays de la Loire & 334 & 30 & 22467 & 4113 & -0.01 & 22470 & 4.26 & 22464 & 3.01 \\
\hline Full instance & 1075 & 35 & 69497 & 5997 & -59.00 & 169968 & 92.80 & 69497 & 5.42 \\
\hline
\end{tabular}

We observe that the heuristic finds very good quality solutions for all instances in two hours of total computation time (heuristic + MIPstart). For the two largest instances, these solutions are far better (around $60 \%$ savings) than the best solution found without heuristic by the MIP in two hours. These solutions are not improved afterwards by CPLEX but using the heuristic as MIPstart enables to obtain the proof of convergence for the two smallest instances and to have all final gaps below $6 \%$.

\section{6 | CONCLUSION}

In this article, we introduced a problem of multi-year investments planning for a telecommunication operator. Encompassing several real aspects faced by operators, our problem consists in optimizing network and subscriber dynamics under capacity and strategic constraints. In particular, we have modeled the fraction of subscribers adopting a new technology as depending on the coverage of that technology. In addition, the operator can provide subsidies to encourage the subscribers to shift faster to that technology. We have provided a non-linear MIP formulation for this problem, which we linearize and reinforce with several sets of valid inequalities. Computational tests have been made for a real 3G/4G case-study. The efficiency of the valid inequalities in improving the performances has been underlined, as well as the relevance of the branch-and-bound procedure performed on the tightened MILP for solving scaled real-life instances. For the largest instance, 
the solver struggles to find a feasible solution and so we have proposed a heuristic to find a goodquality primal feasible solution. This heuristic is based on fixing the upgrade parameters (subsidies amount and state of coverage) and enables us to find very good quality solutions while running much faster than the branch-and-bound procedure on the exact MILP. Our results also illustrate the effect of imposing additional business-oriented constraints needed from the operational viewpoint. For instance, we have modeled the possibility for the operator of smoothing its investments along the time horizon, which enables us to quantify the overcost due to smoothing policies. This overcost is significant, especially in suburban and rural areas. This could push operators to reconsider their investment policies and decide to perform an important initial investment in order to make savings on the whole time horizon.

\section{References}

[1] ARCEP, 2018. Arcep rankings. https://www.arcep.fr/actualites/les-communiques-depresse/detail/n/qualite-des-services-mobiles-1.html (Accessed 10/30/2018).

[2] F.M. Bass, Empirical generalizations and marketing science: A personal view, Mark. Sci. 14 (1995), G6G19.

[3] F.M. Bass, Comments on "a new product growth for model consumer durables the bass model", Manage. Sci. 50 (2004), 1833-1840.

[4] M. Chardy, M.B. Yahia, and Y. Bao, 3g/4g load-balancing optimization for mobile network planning, 2016 17th International Telecommunications Network Strategy and Planning Symposium (Networks), Sept 2016, pp. 7-12.

[5] CISCO, 2017. Cisco visual networking index: Global mobile data traffic forecast update, 2016-2021 white paper. https://www.cisco.com/c/en/us/solutions/collateral/service-provider/visualnetworking-index-vni/mobile-white-paper-c11-520862.html (Accessed 10/30/2018).

[6] DigitallnformationWorld, 2018.. https://www.digitalinformationworld.com/2018/05/infographicinternet-minute-2018.html (Accessed 10/30/2018).

[7] Fortet, Boole algebra and its application to operation research., Trabajos de Estadistica (1960), 111118.

[8] B.L. Garcia, P. Mahey, and L.J. LeBlanc, Iterative improvement methods for a multiperiod network design problem, Eur. J. Oper. Res. 110 (1998), 150-165.

[9] M. Gendreau, J.Y. Potvin, A. Smires, and P. Soriano, Multi-period capacity expansion for a local access telecommunications network, Eur. J. Oper. Res. 172 (2006), 1051-1066.

[10] S. Gollowitzer, L. Gouveia, and I. Ljubic, Enhanced formulations and branch-and-cut for the two level network design problem with transition facilities, Eur. J. Oper. Res. 225 (2013), 211-222.

[11] GSMA, 2020. The mobile economy 2020 (gsm association annual report). https://www.gsma.com/mobileeconomy/wp-content/uploads/2020/03/GSMA_MobileEconomy2020_Global.pdf (Accessed 05/28/2020). 
[12] H. Holma and A. Toskala, LTE Advanced: 3GPP Solution for IMT-Advanced, Wiley Publishing, 1st edition 2012.

[13] A. Knippel and B. Lardeux, The multi-layered network design problem, Eur. J. Oper. Res. 183 (2007), 87-99.

[14] P. Kubat and J.M. Smith, A multi-period network design problem for cellular telecommunication systems, Eur. J. Oper. Res. 134 (2001), 439-456.

[15] D.E. Lim and T. Kim, An application of a multi-generation diffusion model to forecast $5 \mathrm{~g}$ mobile telecommunication service subscribers in south korea, Int. J. Pure Appl. Math. 116 (2017), 809-817.

[16] C. Michalakelis, D. Varoutas, and T. Sphicopoulos, Diffusion models of mobile telephony in greece, Telecommunications Pol. 32 (2008), 234 - 245.

[17] J.A. Norton and F.M. Bass, A diffusion theory model of adoption and substitution for successive generations of high-technology products, Manage. Sci. 33 (1987), 1069-1086.

[18] Orange, 2018. Mobile network worlwide activity (orange group webpage). https://www.orange.com/en/Group/Activities/Networks/Folder/Mobile-network_ (Accessed 10/30/2018).

[19] E. Rogers, Diffusion of Innovations, Simon and Schuster, 5th edition 2003.

[20] P. Sharma, Evolution of mobile wireless communication networks-1g to $5 g$ as well as future prospective of next generation communication network, Int. J. Comput. Sci. Mobile Comput. 2 (2013), 47-53.

[21] H. Sherali and W. Adams, A Reformulation-Linearization Technique for Solving Discrete and Continuous Nonconvex Problems, Nonconvex Optimization and Its Applications, Springer US, 1998. 


\section{Appendix: Extension to more-than-two generations frameworks}

This model can be extended to tackle cases where several generations ( $N \geq 3$ generations) co-exist. Three important questions arise for the operator when extending to more than two generations.

1. It has to decide its network investments policy, i.e. which technology(ies) can be deployed (or not) over the time horizon and for which technologies new modules can be added.

2. It has to define its subsidies policy, i.e. to which current subscriptions and for shifting to which technology(ies) the subsidies are offered. Note that, if allowed by the regulatory context, offered subsidies could have different values according to the current and/or targeted subscription. Finally, we stress the fact that reactions (and thus the modeled upgrade function) could also be different with respect to current and/or targeted technologies.

3. It has to define its load-balancing policy, i.e. which network technology(ies) is/are preferred to serve subscribers of different subscriptions, while respecting technical incompatibilities and deployment of the technology on the associated site. Note that this rule could be no preference among compatible and deployed technologies, a strict priority order (served by the most efficient compatible and deployed technology) or a mix of them (for instance, served by the most efficient and compatible technology if deployed, and no preference among less efficient technologies otherwise).

Let us define the framework where:

1. The operator allows module investments for all technologies, but the deployment of a technology on a site not yet equipped is limited to the newest technology. Besides, we suppose that the oldest generation is deployed everywhere at the beginning of the time horizon.

2. The subsidies are offered to subscribers from current subscriptions (all except the newest one) for shifting to the newest technology only. The subsidies offered to the subscribers and their reaction are considered independent of their initial subscription.

3. Subscribers cannot be served by a more recent technology than the one they have subscribed to (technical incompatibility). In addition, among the compatible technologies, we assume that they have to be served by the most recent and deployed technology (in particular the one they have subscribed to if it has been deployed).

Considering a general set of generations $\mathcal{G}=\left\{c g_{1}, \ldots, c g_{N}, N G\right\}$ of $N$ current generations ordered by efficiency and the newest one NG, the notation proposed in Section 3.1 still hold. The formulation proposed in Section 3.2 can be extended as follows for the framework we have defined:

$$
\begin{aligned}
\min & \sum_{t \in \mathcal{T}} \sum_{\sigma \in \mathcal{K}} \sum_{c \in C} \sum_{s \in \mathcal{S}} \sum_{g \in \mathcal{G} \backslash\{N G\}} \sigma f_{\sigma, c} \pi_{\sigma, c, s, g}^{t} \\
& +\sum_{s \in \mathcal{S}} C A_{N G}\left(z_{s, N G}^{\bar{t}}-Z_{s, N G}^{0}\right)
\end{aligned}
$$$$
+\sum_{s \in \mathcal{S}} \sum_{g \in \mathcal{G}} C M_{g}\left(m_{s, g}^{\bar{t}}-M_{s, g}^{0}\right)
$$ 
s.t. $m_{s, g}^{t} \leq Z_{s, g}^{0} \bar{M}_{g}$

$$
m_{s, N G}^{t} \leq \bar{M}_{N G} z_{s, N G}^{t}
$$

$m_{s, g}^{t-1} \leq m_{s, g}^{t}$

$u_{s, o}^{t}=\sum_{g \in \mathcal{G}: g \leq o} u_{s, o, g}^{t}$

$Z_{s, k}^{0} \sum_{o \in \mathcal{G}: o \geq k} \sum_{g \in \mathcal{G}: g<k} u_{s, o, g}^{t}=0$

$\sum_{g \in \mathcal{G} \backslash\{N G\}} u_{s, N G, g}^{t} \leq \bar{U}_{s, N G}^{t}\left(1-z_{s, N G}^{t}\right)$

$D_{o, g}^{t} \sum_{o \in \mathcal{G}} u_{s, o, g}^{t} \leq C A P_{g} m_{s, g}^{t}$

$u_{s, g}^{t}=u_{s, g}^{t-1}+N_{g}^{t} U T O T_{s}^{t-1}-\sum_{\sigma \in \mathcal{K}} \sum_{c \in C} f_{\sigma, c} \pi_{\sigma, c, s, g}^{t}$

$u_{s, N G}^{t}=u_{s, N G}^{t-1}+N_{N G}^{t} U T O T_{s}^{t-1}+\sum_{g \in \mathcal{G} \backslash\{N G\}} \sum_{\sigma \in \mathcal{K}} \sum_{c \in C} f_{\sigma, c} \pi_{\sigma, c, s, g}^{t} \quad \forall s \in \mathcal{S}, \forall t \in \mathcal{T}$,

$\sum_{s \in \mathcal{S}} u_{s, N G, N G}^{\bar{t}} \geq \sum_{s \in \mathcal{S}} \underline{Q \circ E} U_{T O T_{s}^{t}}$

$\alpha^{\bar{t}} \geq \underline{\alpha}$

$\sum_{\sigma \in \mathcal{K}} \sum_{c \in \mathcal{C}} \delta_{\sigma, c}^{t}=1$

$\sum_{\sigma \in \mathcal{K}} \delta_{\sigma, c}^{t} \leq 1+U_{c}-\alpha^{t-1}$

$\sum_{\sigma \in \mathcal{K}} \delta_{\sigma, c}^{t} \leq 1+\alpha^{t-1}-L_{c}$

$\pi_{\sigma, c, s, g}^{t} \leq \delta_{\sigma, c}^{t} \bar{U}_{s, g}^{t-1}$

$\pi_{\sigma, c, s, g}^{t} \leq u_{s, g}^{t-1}$

$\pi_{\sigma, c, s, g}^{t} \geq u_{s, g}^{t-1}-\left(1-\delta_{\sigma, c}^{t}\right) \bar{U}_{s, g}^{t-1}$

$u_{s, g}^{0}=U_{s, g}^{0}$

$m_{s, g}^{0}=M_{s, g}^{0}$

$z_{s, N G}^{0}=Z_{s, N G}^{0}$ $\forall s \in \mathcal{S}, \forall t \in \mathcal{T}, \forall g \in \mathcal{G} \backslash\{N G\}$,

$\forall s \in \mathcal{S}, \forall t \in \mathcal{T}$,

$\forall s \in \mathcal{S}, \forall t \in \mathcal{T}, \forall g \in \mathcal{G}$,

$\forall s \in \mathcal{S}, \forall t \in \mathcal{T}, \forall 0 \in \mathcal{G}$,

$\forall s \in \mathcal{S}, \forall t \in \mathcal{T}, \forall k \in \mathcal{G} \backslash\{N G\}$

$\forall s \in \mathcal{S}, \forall t \in \mathcal{T}$,

$\forall s \in \mathcal{S}, \forall t \in \mathcal{T}, \forall g \in \mathcal{G}$,

$\forall s \in \mathcal{S}, \forall t \in \mathcal{T}, \forall g \in \mathcal{G} \backslash\{N G\}$,

$\forall t \in \mathcal{T}, \forall c \in C$,

$\forall t \in \mathcal{T}, \forall c \in C$,

$\forall s \in \mathcal{S}, \forall t \in \mathcal{T}, \forall \sigma \in \mathcal{K}, \forall c \in \mathcal{C}, \forall g \in \mathcal{G}$

$\forall s \in \mathcal{S}, \forall t \in \mathcal{T}, \forall \sigma \in \mathcal{K}, \forall c \in \mathcal{C}, \forall g \in \mathcal{G}$,

$\forall s \in \mathcal{S}, \forall t \in \mathcal{T}, \forall \sigma \in \mathcal{K}, \forall c \in \mathcal{C}, \forall g \in \mathcal{G}$,

$\forall s \in \mathcal{S}, \forall g \in \mathcal{G}$,

$\forall s \in \mathcal{S}, \forall g \in \mathcal{G}$,

$\forall s \in \mathcal{S}$, 


$$
\begin{aligned}
& m_{s, g}^{t} \in \mathbb{N} \\
& z_{s, N G}^{t} \in\{0,1\} \\
& u_{s, g}^{t} \geq 0 \\
& u_{s, o, g}^{t} \geq 0 \\
& \delta_{\sigma, c}^{t} \in\{0,1\} \\
& \pi_{\sigma, c, s, g}^{t} \in\{0,1\}
\end{aligned}
$$$$
\forall s \in \mathcal{S}, \forall t \in \mathcal{T} \cup\{0\}, \forall g \in \mathcal{G},
$$$$
\forall s \in \mathcal{S}, \forall t \in \mathcal{T} \cup\{0\},
$$$$
\forall s \in \mathcal{S}, \forall t \in \mathcal{T} \cup\{0\}, \forall g \in \mathcal{G},
$$$$
\forall s \in \mathcal{S}, \forall t \in \mathcal{T}, \forall o, g \in \mathcal{G}^{2},
$$$$
\forall t \in \mathcal{T}, \forall \sigma \in \mathcal{K}, \forall c \in C
$$

$\forall s \in \mathcal{S}, \forall t \in \mathcal{T}, \forall \sigma \in \mathcal{K}, \forall c \in C, \forall g \in \mathcal{G}$

The objective function 81 minimizes both subscribers migration costs and network investments. The first term stands for the subsidies offered (user upgrades); the second term for the adding of new modules for increasing the capacity (densification); and the third term for the deployment of the newest technology $N G$ (coverage extension).

Constraints [82 - 84] are the network dynamic constraints. Constraints [82 -83] define the upper bounds on the numbers of modules for each technology added on each site. These constraints also ensure that if a technology is not deployed, no corresponding modules can be added. Constraints 84 prevent from decommissioning by imposing the number of modules of each technology to be non-decreasing during the time horizon.

Constraints 85, 88 are the network dimensioning constraints, in charge of making the link between the network and the subscriber dynamic. Constraints 85 ensure the technical incompatibility stating that subscribers cannot be served by a more recent technology than the one they have subscribed to. Constraints 86 and 87 ] ensure the load-balancing rule. Constraints 88 are the capacity constraints: the installed capacities of each technology on each site have to be sufficient for providing services for all users located at this site and having to be served by this technology.

Constraints 89-90] are the subscriber dynamic constraints. They define the total number of subscribers to each technology at each site and each time period, taking into account subscribers to current generations who decide to upgrade to $N G$ technology, thanks to subsidies and coverage improvements. Constraints 91, 92 stand for the model strategic guidelines and refer to the end of time horizon. Constraint 91] ensures the threshold of subscribers covered by the newest technology is met. The indicator is proportional to the quality of experience which measures the percentage of users having access to the new technology throughput. Constraint 92] imposes that the threshold on the number of sites on which $N G$ is deployed is met.

Constraints 93. ensure that one and only one subsidy from the set $\mathcal{K}$ is offered at each time period, the case when no subsidy is given being represented by $\sigma=0$. Constraints 94 and 95] ensure that, for each time period, variables $\delta_{\sigma, c}^{t}$ are set according to the coverage at the previous time 
period. Constraints 94 (respectively 95) set all $\delta$ related to a range at 0 if the coverage is greater (resp. smaller) than the upper (resp. lower) bound of the range. Constraints 96- 98) are the typical linearizations of the products of a binary variable with a continuous one. Constraints 99- 101 . refer to the initial conditions. Finally, constraints 102 - 107 define the domain of the variables.

Remark 15 The remark 2 on variables $m$ still holds. 